\title{
Scutellarin Suppresses NLRP3 Inflammasome Activation in Macrophages and Protects Mice against Bacterial Sepsis
}

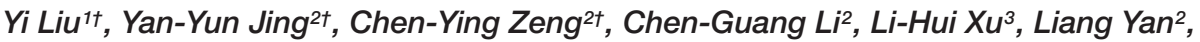 \\ Wen-Jing Bai ${ }^{2}$, Qing-Bing Zha ${ }^{4}$, Dong-Yun Ouyang ${ }^{2}$ and Xian-Hui He ${ }^{2 *}$
}

${ }^{1}$ Institute of Dermatology, Chinese Academy of Medical Sciences, Nanjing, China, ${ }^{2}$ Department of Immunobiology, College of Life Science and Technology, Jinan University, Guangzhou, China, ${ }^{3}$ Department of Cell Biology, College of Life Science and Technology, Jinan University, Guangzhou, China, ${ }^{4}$ Department of Fetal Medicine, The First Affiliated Hospital of Jinan University, Guangzhou, China

\section{OPEN ACCESS}

Edited by:

Suresh Kumar,

Universiti Putra Malaysia, Malaysia

Reviewed by:

Subhalakshmi Ghosh,

Jadavpur University, India

Ouyang Chen,

Second Military Medical University,

China

Mingliang Cheng,

Guiyang Medical University, China

*Correspondence:

Xian-Hui He

thexh@jnu.edu.cn

${ }^{\dagger}$ These authors have contributed equally to this work.

Specialty section:

This article was submitted to

Ethnopharmacology,

a section of the journal

Frontiers in Pharmacology

Received: 02 September 2017

Accepted: 20 December 2017

Published: 09 January 2018

Citation:

Liu Y, Jing Y-Y, Zeng C-Y, Li C-G,

$X u$ L-H, Yan L, Bai W-J, Zha Q-B,

Ouyang D-Y and He X-H (2018)

Scutellarin Suppresses NLRP3

Inflammasome Activation

in Macrophages and Protects Mice

against Bacterial Sepsis.

Front. Pharmacol. 8:975.

doi: 10.3389/fphar.2017.00975
The NLRP3 inflammasome plays a critical role in mediating the innate immune defense against pathogenic infections, but aberrant activation of NLRP3 inflammasome has been linked to a variety of inflammatory diseases. Thus targeting the NLRP3 inflammasome represents a promising therapeutic for the treatment of such diseases. Scutellarin is a flavonoid isolated from Erigeron breviscapus (Vant.) Hand.-Mazz. and has been reported to exhibit potent anti-inflammatory activities, but the underlying mechanism is only partly understood. In this study, we aimed to investigate whether scutellarin could affect the activation of NLRP3 inflammasome in macrophages. The results showed that scutellarin dose-dependently reduced caspase- 1 activation and decreased mature interleukin-1 $\beta$ $(\mathrm{IL}-1 \beta)$ release in lipopolysaccharide (LPS)-primed macrophages upon ATP or nigericin stimulation, indicating that scutellarin inhibited NLRP3 inflammasome activation in macrophages. Consistent with this, scutellarin also suppressed pyroptotic cell death in LPS-primed macrophages treated with ATP or nigericin. ATP or nigericin-induced ASC speck formation and its oligomerization were blocked by scutellarin pre-treatment. Intriguingly, scutellarin augmented PKA-specific phosphorylation of NLRP3 in LPSprimed macrophages, which was completely blocked by selective PKA inhibitor H89, suggesting that PKA signaling had been involved in the action of scutellarin to suppress NLRP3 inflammasome activation. Supporting this, the inhibitory effect of scutellarin on NLRP3 inflammasome activation was completely counteracted by H89 or adenyl cyclase inhibitor MDL12330A. As NLRP3-dependent release of IL-1 $\beta$ has a critical role in sepsis, the in vivo activity of scutellarin was assayed in a mouse model of bacterial sepsis, which was established by intraperitoneally injection of a lethal dose of viable Escherichia coli. Oral administration of scutellarin significantly improved the survival of mice with bacterial sepsis. In line with this, scutellarin treatment significantly reduced serum $\mathrm{IL}-1 \beta$ levels and attenuated the infiltration of inflammatory cells in the liver of E. coli-infected mice. These data indicated that scutellarin suppressed NLRP3 inflammasome activation in macrophages by augmenting PKA signaling, highlighting its potential therapeutic application for treating NLRP3-related inflammatory diseases.

Keywords: scutellarin, NLRP3 inflammasome, pyroptosis, protein kinase A, macrophages 


\section{INTRODUCTION}

The NLRP3 (NOD-like receptor family, pyrin domain containing 3; also called cryopyrin and NALP3) is an intracellular sensor that can be activated by a diverse array of factors derived from pathogens, environments and hosts (Guo et al., 2015; Broz and Dixit, 2016; He et al., 2016; Jo et al., 2016; Rathinam and Fitzgerald, 2016). Upon activation, NLRP3 can recruit the adaptor ASC (apoptosis-associated speck-like protein containing a caspase recruitment domain) and procaspase- 1 to form a large multiprotein complex named inflammasome, leading to the autocatalytic activation of caspase- 1 . Subsequently, active caspase- 1 in turn converts pro-interleukin (IL)- $1 \beta$ and pro-IL-18 into their mature forms, which are released into the extracellular compartments and act as potent proinflammatory cytokines ( $\mathrm{He}$ et al., 2016; Jo et al., 2016). Concurrently, active caspase-1 also cleaves gasdermin $\mathrm{D}$ to release its active $\mathrm{N}$-terminal fragment which form pores on the plasma membrane thus culminating in pyroptotic cell death and the release of proinflammatory intracellular components into the extracellular milieu (Ding et al., 2016; Liu et al., 2016; Shi et al., 2017). Thus, during pathogenic infections, NLRP3 inflammasomes represent highly proinflammatory platforms that play critical roles in clearing microbial infection and alerting the immune system (de TorreMinguela et al., 2017).

Macrophages have an important role in mediating the inflammatory responses during infections. Two signals are required to activate the NLRP3 inflammasome in macrophages (He et al., 2016; Jo et al., 2016): The first signal (signal 1) induces the expression of critical inflammasome components including NLRP3 and pro-IL-1 $\beta$; this signal is mediated by pattern recognition receptors (PRRs) through pathogenassociated molecular patterns (PAMPs). Once such proteins are sufficiently accumulated, the second signal (signal 2) is needed to trigger the assembly of NLRP3 inflammasome. Lipopolysaccharide (LPS) is commonly used PAMPs to confer signal 1, while DAMPs like extracellular ATP (Mariathasan et al., 2006) can act as signal 2 to trigger NLRP3 inflammasome activation in LPS-primed macrophages. ATP can be released from bacteria or host cells during bacterial infections or sterile tissue damages (Piccini et al., 2008; Wegiel et al., 2014), thus being an important triggering signal for NLRP3 inflammasome activation.

Although NLRP3 inflammasomes play critical role in combating against infections, aberrant activation of this inflammasome has been linked to many inflammatory diseases (Guo et al., 2015; de Torre-Minguela et al., 2017). Excessive NLRP3 inflammasome activation results in robust and rapid inflammation due to the release of many proinflammatory cytokines including IL-1 $\beta$. These massive proinflammatory cytokines in turn enhance pyroptosis leading to multiple organ damage and septic death during bacterial infections (Wree et al., 2014; Kayagaki et al., 2015). In support of this notion, hyperactive NLRP3 due to conditional NLRP3 mutant knock-in culminates in increased hepatocyte pyroptosis and liver injury with decreased mouse survival (Wree et al., 2014). On the other hand, blockade of pyroptosis by caspase-1/-11 or gasdermin $\mathrm{D}$ gene deletion renders mice resistant to endotoxin-induced sepsis (Kayagaki et al., 2011, 2015), suggesting NLRP3 and other inflammasome activation play a critical role in sepsis. Excessive activation of NLRP3 inflammasome has also been linked to a variety of inflammatory disorders or metabolic disorders, such as Alzheimer's disease, atherosclerosis, gout, and obesity (Wen et al., 2012). Intriguingly, NLRP3 depletion dampens the severity of atherosclerosis (Duewell et al., 2010), multiple sclerosis (Shaw et al., 2010), Alzheimer's disease (Heneka et al., 2013), type 2 diabetes (Vandanmagsar et al., 2011; Wen et al., 2011), and gout (Martinon et al., 2006). Thus, agents targeting NLRP3 activation are promising candidates for the treatment of NLRP3-related diseases.

Scutellarin $\quad\left(4^{\prime}, 5,6\right.$-hydroxyl-flavone-7-glucuronide $)$ is a flavonoid purified from Erigeron breviscapus (Vant.) Hand.Mazz. (Gao et al., 2007). This agent has been extensively investigated for its anti-inflammatory and neuroprotective activities (Yuan et al., 2016; Gao et al., 2017). Several studies showed that scutellarin inhibits the expression of inflammatory cytokines, such as tumor necrosis factor (TNF)- $\alpha$ and IL-6, through suppressing the NF-KB pathway in LPS-activated microglia (Wang et al., 2011; Chen et al., 2013) as well as in LPS-induced acute lung injury (Tan et al., 2010). Consistent with these studies, a separate report also showed that oral administration scutellarin significantly attenuated neurological injury in a rat model of ischemia injury, accompanied by reduced expression of TNF- $\alpha$, IL-1 $\beta$, and IL- 6 (Wang W. et al., 2016). By suppressing the NF- $\mathrm{KB}$ and mitogen-activated protein kinase pathways, scutellarin can inhibit RANKL-mediated osteoclastogenesis and titanium particle-induced osteolysis (Zhao et al., 2016). Beyond the NF- $\kappa B$ signaling, the Notch pathway has been identified to be involved in the action of scutellarin on microglia activation (Yuan et al., 2015). Scutellarin has also been shown to attenuate diosbulbin B-induced liver injury by suppressing proinflammatory cytokines (Niu et al., 2015). In clinical practice, scutellarin has been used for the treatment of ischemic cardiovascular and cerebrovascular diseases (Yuan et al., 2016; Gao et al., 2017). The protective effects of scutellarin against hypoxic-ischemic cardiomyocyte and brain injury have been shown to be mediated by its antioxidant capacity (Guo et al., 2011; Wang Z. et al., 2016). As ischemic cerebrovascular diseases involve brain injury and injury-related inflammatory responses (sterile inflammatory responses), it has been proposed that scutellarin may confer neuroprotection by suppressing the activation of microglia (Wang et al., 2011; Yuan et al., 2014, 2015). It is worth noting that during brain injury, endogenous danger signals including ATP are released into the extracellular milieu to activate NLRP3 inflammasomes, thus further exacerbating the injury-related inflammatory responses (Ito et al., 2015). However, it remains unknown whether scutellarin has any influences on the activation of NLRP3 inflammasome in the innate immune cells including macrophages. Its effects on NLRP3-related inflammatory diseases including sepsis also await clarification.

In this study, we found that scutellarin inhibited NLRP3 inflammasome activation and pyroptosis in LPS-primed macrophages upon ATP or nigericin stimulation. ASC speck formation and oligomerization were robustly suppressed by 
scutellarin. Such scutellarin-mediated suppression of NLRP3 activation and ASC speck formation was abrogated by the protein kinase A (PKA) pathway inhibitors, indicating the involvement of PKA signaling in this process. In further support of this, the Ser/Thr phosphorylation of NLRP3 on PKA-specific sites was markedly increased by scutellarin but was blocked by PKA inhibitor H89. Besides, oral administration of scutellarin significantly attenuated systemic inflammation and improved the survival of mice with bacterial sepsis, suggesting that it could inhibit NLRP3 activation in vivo. Our data reveals that scutellarin is able to suppress NLRP3 inflammasome activation thus exhibiting therapeutic effects against NLRP3-related inflammatory diseases.

\section{MATERIALS AND METHODS}

\section{Reagents}

Scutellarin ( $\geq 98 \%$ purity) (110842) was obtained from Guangdong Institute for Drug Control (Guangzhou, China). Dulbecco's Modified Eagle's Medium (DMEM) medium with high glucose, Opti-MEM, fetal bovine serum (FBS), streptomycin and penicillin were products of ThermoFisher/Gibco (Carlsbad, CA, United States). MDL12330A (M182), adenosine triphosphate (ATP) (A6419), lipopolysaccharide (LPS) (Escherichia coli O111:B4) (L4391), dimethyl sulfoxide (DMSO) (D8418), propidium iodide (P4170), Hoechst 33342 (B2261), Tween 80 (P8074) and disuccinimidyl suberate (S1885) were purchased from Sigma-Aldrich (St. Louis, MO, United States). H89 (S1643), cell lysis buffer for Western and IP (P0013) and phenylmethanesulfonyl fluoride (PMSF) (ST505) were bought from Beyotime Biotechnology (Haimen, China). Nigericin (\#tlrl-nig) was purchased from InvivoGen (San Diego, CA, United States). The antibody against caspase-1p10 (sc-514) was purchased from Santa Cruz (Dallas, TX, United States). The antibody against NLRP3 (Cryo-2) (AG-20B-0014) was purchased from Adipogen AG (Liestal, Switzerland). Specific antibodies against IL-1 $\beta$ (\#12242), HMGB1 (\#3935), ASC (\#67824), p-(Ser/Thr) PKA substrate (\#9621), and $\beta$-tubulin (\#2128) were bought from Cell Signaling Technology (Danvers, MA, United States). The horse-radish peroxidase (HRP)conjugated horse anti-mouse IgG (\#7076), HRP-conjugated goat-anti-rabbit IgG (\#7074) and Protein G Agarose beads (\#37478) were also from Cell Signaling Technology. CF568 goat-anti-rabbit IgG $(\mathrm{H}+\mathrm{L})$, highly cross-adsorbed (\#20103) and CF488A-conjugated goat-anti-mouse IgG (\#20018), highly cross-adsorbed were obtained from Biotium (Hayward, CA, United States). Scutellarin was dissolved in DMSO at $100 \mathrm{mM}$, and stored at $-20^{\circ} \mathrm{C}$.

\section{Experimental Mice}

Female C57BL/6 mice (6-8 weeks of age) were purchased from the Experimental Animal Center of Southern Medical University (Guangzhou, China). Mice were acclimatized for 1 week before experiments. All animal experiments were performed in accordance with the guidelines for the care and use of animals approved by the Committee on the Ethics of Animal Experiments of Jinan University (approved No. JNU20160315).

\section{Macrophage Culture}

Bone marrow-derived macrophages (BMDMs) were differentiated as described previously (Kayagaki et al., 2011; Li et al., 2017). In brief, C57BL/6 mice were sacrificed and bone marrow cells were differentiated in culture medium (DMEM supplemented with $10 \%$ FBS, $100 \mathrm{U} / \mathrm{ml}$ penicillin, $100 \mu \mathrm{g} / \mathrm{ml}$ streptomycin and 20\% M-CSF-conditioned medium) for 6 days at $37^{\circ} \mathrm{C}$ in a humidified incubator of $5 \% \mathrm{CO}_{2}$. BMDMs were cultured in 24 -well plates at $1.5 \times 10^{5}$ cells/well $(0.5 \mathrm{ml})$ or in 6 -well plates at $1.2 \times 10^{6}$ cells/well $(2 \mathrm{ml})$ or in $10-\mathrm{cm}$ petri dish at $7.5 \times 10^{6}$ cells/dish $(10 \mathrm{ml})$ with complete DMEM medium (DMEM supplemented with $10 \% \mathrm{FBS}, 100 \mathrm{U} / \mathrm{ml}$ penicillin, and $100 \mu \mathrm{g} / \mathrm{ml}$ streptomycin) at $37^{\circ} \mathrm{C}$ overnight.

\section{Cell Death Assay}

Cell death was measured by propidium iodide (PI) incorporation as described previously (Py et al., 2014; Li et al., 2017). Briefly, BMDMs were primed in Opti-MEM with $500 \mathrm{ng} / \mathrm{ml}$ LPS for $4 \mathrm{~h}$. Then the cells were treated with different doses of scutellarin for $1 \mathrm{~h}$ followed by ATP $(3 \mathrm{mM})$ or nigericin $(10 \mu \mathrm{M})$ for indicated time periods. The nuclei were revealed by Hoechst $33342(5 \mu \mathrm{g} / \mathrm{ml})$ staining and dead cells revealed by PI $(2 \mu \mathrm{g} / \mathrm{ml})$ staining at room temperature for $10 \mathrm{~min}$. The cells were observed immediately by live imaging using Zeiss Axio Observer D1 microscope equipped with a Zeiss LD PlanNeofluar $20 \times / 0.4$ Korr M27 objective lens (Carl Zeiss MicroImaging GmbH, Göttingen, Germany). Fluorescence images were captured with a Zeiss AxioCam MR R3 cooled CCD camera controlled with ZEN software (Carl Zeiss).

\section{Precipitation of Soluble Proteins}

Soluble protein in culture supernatants was precipitated as previously described (Kayagaki et al., 2011; Li et al., 2017). Briefly, soluble proteins in culture supernatants (equal volume for each sample) were precipitated with $7.2 \%$ trichloroacetic acid plus $0.15 \%$ sodium deoxycholate. The precipitated proteins were re-dissolved in $2 \times$ sodium dodecylsulfate-polyacrylamide gel electrophoresis (SDS-PAGE) sample loading buffer and subjected to western blot analysis for released mature IL- $1 \beta$, caspase-1p10 and HMGB1.

\section{Immunoblotting}

Immunoblotting was performed essentially as previously described (Lin et al., 2016). In brief, equal amounts of proteins were separated by SDS-PAGE followed by electrotransfer to polyvinylidene difluoride (PVDF) membranes (\#03010040001; Roche Diagnostics GmbH, Mannheim, Germany). Membranes were blocked and incubated with indicated primary antibodies at $4^{\circ} \mathrm{C}$ overnight, followed by incubation with appropriate HRP-conjugated secondary antibody. Bands were revealed by a BeyoECL Plus kit (P0018; Beyotime, Haimen China) and recorded on X-ray films (Carestream, Xiamen, China). The densitometry of each band was quantified by FluorChem 8000 (Alpha Innotech; San Leandro, CA, United States). 


\section{Soluble IL-1 $\beta$ Detection}

Soluble cytokine IL-1 $\beta$ in culture supernatants of BMDMs was detected by Cytometric Bead Array (CBA) Mouse IL-1 $\beta$ Flex Set (BD Biosciences, San Jose, CA, United States) according to the manufacturer's instructions. Data were acquired on a flow cytometer (FACSCalibur; Becton Dickinson) equipped with CELLQuest Pro software (Becton Dickinson).

\section{Immunofluorescence Microscopy}

Immunofluorescence analysis was performed as previously described (Zhao et al., 2015; Zha et al., 2016). Briefly, BMDMs were seed in glass-bottomed dishes $\left(4 \times 10^{5}\right.$ cells/dish $)$ and cultured in complete DMEM medium at $37^{\circ} \mathrm{C}$ overnight. Cells were primed in Opti-MEM with $500 \mathrm{ng} / \mathrm{ml}$ LPS for $4 \mathrm{~h}$, and then treated with different doses of scutellarin for $1 \mathrm{~h}$ followed by treatment with $3 \mathrm{mM}$ ATP for $30 \mathrm{~min}$ or $10 \mu \mathrm{M}$ nigericin for $1 \mathrm{~h}$ in Opti-MEM. After fixation, permeabilization and blocking, the cells were incubated with anti-ASC antibody (1:300) and anti-NLRP3 antibody (1:300) overnight at $4^{\circ} \mathrm{C}$, followed by staining with CF568-conjugated goat-anti-rabbit IgG and CF488A-conjugated goat-anti-mouse IgG. Nuclei were revealed by Hoechst 33342 and ASC speck formation was observed under a Zeiss Axio Observer D1 microscope with a Zeiss LD Plan-Neofluar 40×/0.6 Korr M27 objective (Carl Zeiss MicroImaging GmbH, Göttingen, Germany). Fluorescence images were captured by a Zeiss AxioCam MR R3 cooled CCD camera controlled with ZEN software (Carl Zeiss).

\section{ASC Oligomerization}

The cross-linking of ASC oligomers was performed as described previously (He et al., 2014; Coll et al., 2015). Briefly, BMDMs were seeded in 6-well plates at $1.0 \times 10^{6}$ cells/well. After appropriate treatments, cells were lysed with cold PBS containing $0.5 \%$ Triton $\mathrm{X}-100$, and the cell lysates were centrifuged at $6000 \times g$ for $15 \mathrm{~min}$ at $4^{\circ} \mathrm{C}$. The pellets were washed twice with PBS and then re-suspended in $200 \mu \mathrm{l}$ PBS. Freshly prepared disuccinimidyl suberate $(2 \mathrm{mM})$ was added to the re-suspended pellets and the suspension was incubated at room temperature for $30 \mathrm{~min}$ with rotation. The cross-linked pellets were collected by centrifugation at $6000 \times \mathrm{g}$ for $15 \mathrm{~min}$ at $4^{\circ} \mathrm{C}$ and re-dissolved in $25 \mu \mathrm{l}$ of $1 \times$ SDS-PAGE sample loading buffer. Samples were boiled for $5 \mathrm{~min}$ and subjected to western blot analysis.

\section{Immunoprecipitation}

After appropriate treatments, cells were rinsed once with ice-cold PBS and lyzed with $0.5 \mathrm{ml}$ ice-cold cell lysis buffer for Western blot and IP (containing $1 \mathrm{mM}$ PMSF). Cell lysates were centrifuged at $13,000 \times g$ for $10 \mathrm{~min}$ at $4^{\circ} \mathrm{C}$ and pre-cleared with a $10 \%$ volume of Protein G agarose beads for $30 \mathrm{~min}$ at $4^{\circ} \mathrm{C}$ with gentle agitation. The pre-cleared cell lysates were then incubated with anti-NLRP3 antibody (1:200) overnight at $4^{\circ} \mathrm{C}$ with gentle shaking. Antibody-NLRP3 complexes were collected with a $10 \%$ volume of Protein $\mathrm{G}$ agarose beads for $2 \mathrm{~h}$ at $4^{\circ} \mathrm{C}$ with gentle shaking. The beads were washed five times with cell lysis buffer, boiled for $5 \mathrm{~min}$ in $3 \times$ SDS-PAGE sample loading buffer, and resolved by Western blot analysis.

\section{Bacterial Infection}

The murine model of bacterial sepsis was established according to the protocol described previously (Wegiel et al., 2014; Zha et al., 2016). In brief, thirty C57BL/6 mice were acclimated for 1 week, randomly divided into three groups (10 mice/group), and intragastrically administered once with scutellarin solution (100 mg/kg or $200 \mathrm{mg} / \mathrm{kg}$ body weight) or vehicle (2\% Tween-80 in PBS), which has referred to previous studies (Li et al., 2011; Yuan et al., 2014; Wang W. et al., 2016). Three hour later, viable E. coli $(\mathrm{DH} 5 \alpha)$ cells $\left(2.5 \times 10^{9}\right.$ colony-forming units $(\mathrm{CFU}) /$ mouse, in $0.5 \mathrm{ml}$ of PBS) were injected into the peritoneal cavity of each mouse. One hour after bacterial infection, mice were intragastrically administered once again with scutellarin solution or vehicle, respectively. Mouse survival was monitored every $6 \mathrm{~h}$ for 5 consecutive days.

In another experiment, 54 mice (6 mice/group) were treated with scutellarin (100 or $200 \mathrm{mg} / \mathrm{kg}$ body weight) or vehicle once by oral gavage, respectively. Three hour later, viable E. coli cells $\left(2.0 \times 10^{9} \mathrm{CFU} /\right.$ mouse, in $0.5 \mathrm{ml}$ of $\left.\mathrm{PBS}\right)$ were injected into the peritoneal cavity of all mice. One hour later, mice were gavaged once again with scutellarin or vehicle, respectively. The mice were sacrificed 4 and $8 \mathrm{~h}$ after bacterial infection, respectively. Their sera were collected, and serum IL- $1 \beta$ levels were measured by CBA mouse IL- $1 \beta$ Flex Set. The bacterial loads were measured as described previously (Pan et al., 2015). The liver and intestine were isolated and fixed in $4 \%$ neutral formaldehyde. Paraffin slices of the tissues were stained with hematoxylin and eosin. Images were captured under the Zeiss Axio Observer D1 microscope armed with a color CCD (ZEISS).

\section{Statistical Analysis}

Experiments were performed three times independently, with one representative experiment shown. Data were expressed as mean \pm standard deviation (SD). Statistical analysis was performed using GraphPad Prism5.0 (GraphPad Software Inc, San Diego, CA, United States). One-way analysis of variance (ANOVA) followed by Tukey post hoc test and unpaired Student's $t$-test were used to analyze the statistical significance among multiple groups and between two groups, respectively. Kaplan-Meier survival curves were used for analysis of mouse survival, and the statistical difference between 2 groups was determined using the log-rank (Mantel-Cox) test. $P$-value $<0.05$ was considered statistically significant.

\section{RESULTS}

\section{Scutellarin Suppressed ATP or Nigericin-Induced Caspase-1 Activation and IL-1 $\beta$ Secretion in Macrophages}

By using bone marrow-derived macrophages (BMDMs) primed with LPS, we initially sought to explore the influence of scutellarin on NLRP3 inflammasome activation upon canonical NLRP3 activator stimulation. Western blot analysis showed that ATP treatment of LPS-primed macrophages 

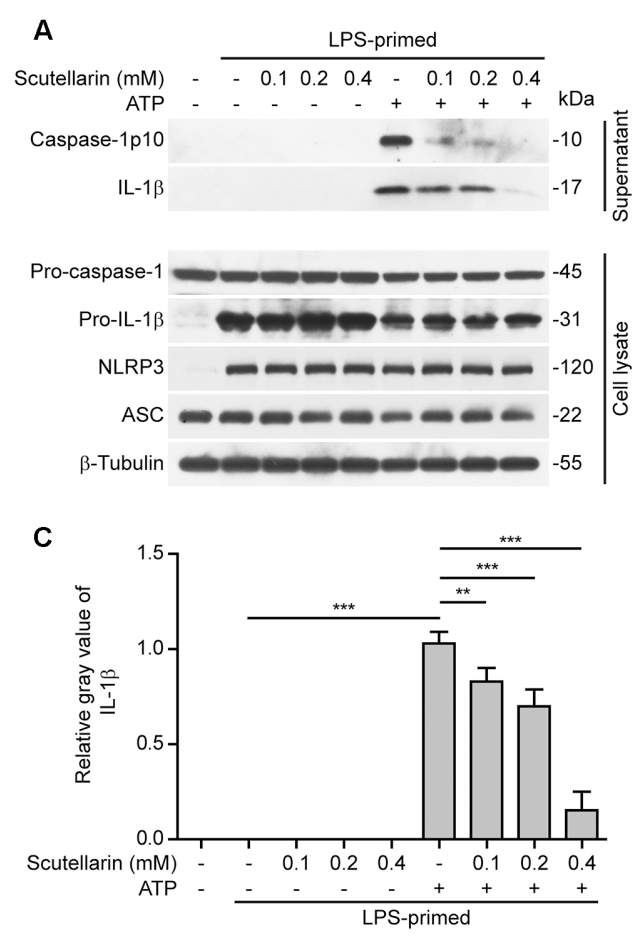

E

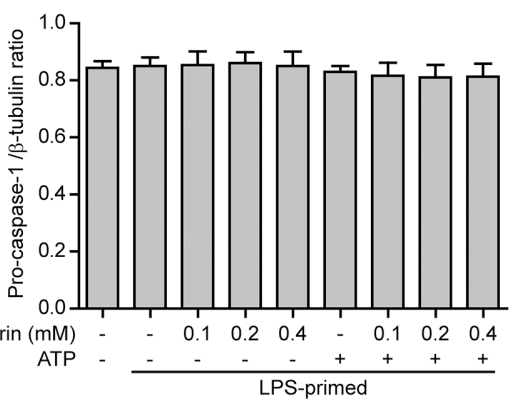

G

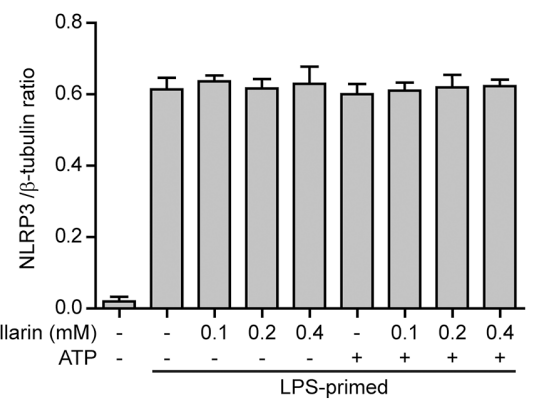

B
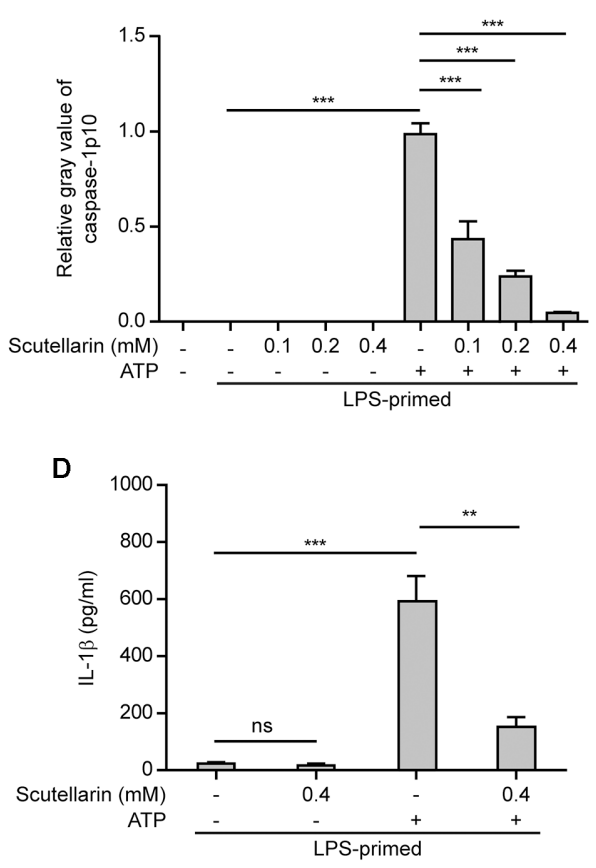

$\mathbf{F}$
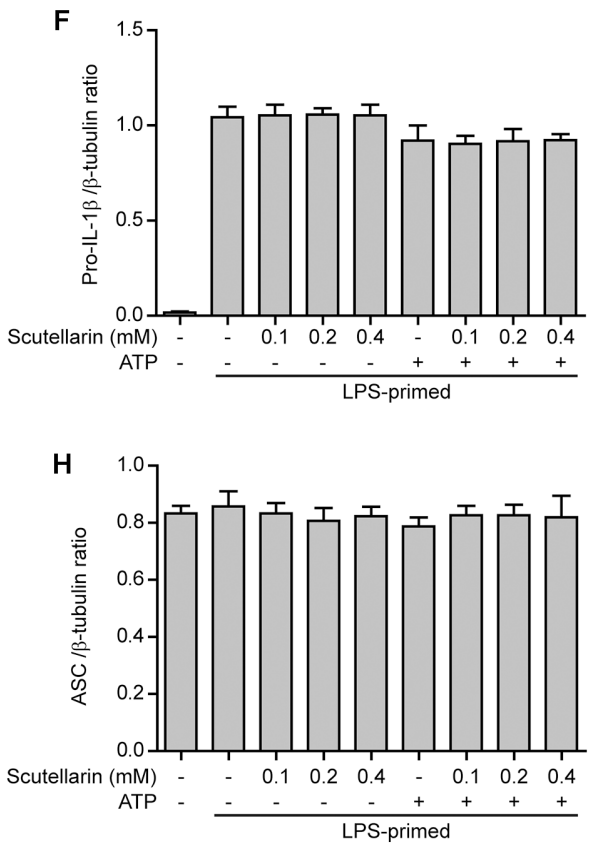

FIGURE 1 | Scutellarin inhibited ATP-induced caspase-1 activation and mature IL-1 $\beta$ secretion in macrophages. Bone marrow-derived macrophages (BMDMs) were first primed with LPS $(500 \mathrm{ng} / \mathrm{ml}$ ) for $4 \mathrm{~h}$, and then pre-treated with indicated concentration of scutellarin for $1 \mathrm{~h}$, followed by stimulation with ATP (3 mM) for 30 min without LPS. (A) Western blot analysis of caspase- 1 and IL-1 $\beta$ in the culture supernatants and indicated proteins in cell lysates. $\beta$-Tubulin was used as a loading control for cell lysates. See Supplementary Material for source blots. (B,C) Histograms showing the relative gray values of capase-1p10 (B) or mature IL-1 $\beta \mathbf{( C )}$ in (A). The gray value of capase-1p10 or IL-1 $\beta$ bands in ATP group was set to 1.0 with those of the other groups being calculated relatively to the ATP group. (D) Cells were treated as in (A). Cytometric bead array (CBA) was used to assay $\mathrm{LL}-1 \beta$ levels in the culture supernatants. Data are shown as mean $\pm \mathrm{SD}(n=3)$. (E-H) Histograms showing the ratios of gray values of indicated proteins relative to $\beta$-tubulin in (A) $(n=3) .{ }^{* *} P<0.01$; ${ }^{* * *} P<0.001$; ns, not significant.

induced a rapid release of active caspase-1p10 (10 kDa) and mature IL-1 $\beta \quad(17 \mathrm{kDa})$ into culture supernatants, indicative of NLRP3 inflammasome activation. Notably, scutellarin pre-treatment dose-dependently reduced ATPinduced release of active caspase-1p10 and mature IL-1 $\beta$ from macrophages, whereas scutellarin per se did not induce 
the release of these proteins (Figures 1A-C). Consistent with previous studies (Kayagaki et al., 2013), the pro-IL$1 \beta$ and NLRP3 proteins were highly expressed after LPS priming, whereas pro-caspase-1 and ASC were constitutively expressed regardless of LPS priming in BMDMs (Figure 1A). We also detected the mature IL-1 $\beta$ levels in the culture supernatants by using a bead-based immunoassay (CBA), and found that scutellarin markedly reduced IL- $1 \beta$ release upon ATP stimulation (Figure 1D), thus confirming the result of western blot analysis of IL- $1 \beta$ release (Figure 1A). Besides, scutellarin had minimal effects on the expression of pro-caspase-1, pro-IL-1 $\beta$, NLRP3, and ASC in the cell lysates (Figures 1A,E-H). These results indicated that scutellarin inhibited NLRP3 inflammasome activation in macrophages upon ATP treatment.

Apart from extracellular ATP, nigericin is another commonly used activator for the NLRP3 inflammasome ( $\mathrm{He}$ et al., 2016). Different from ATP that acts on the plasma membrane P2X7 purinergic receptor to induce $\mathrm{K}^{+}$efflux thus triggering NLRP3 activation, nigericin is a potassium ionophore thus bypassing the $\mathrm{P} 2 \mathrm{X} 7$ receptor to trigger NLRP3 inflammasome activation (Mariathasan et al., 2006). Similar to those results obtained from ATP-treated macrophages (Figure 1), scutellarin dose-dependently reduced caspase-1p10 and mature IL-1 $\beta$ secretion from LPS-primed macrophages upon nigericin stimulation (Figures 2A-D), suggesting that it suppressed nigericin-induced NLRP3 activation. Different from ATP stimulation, under nigericin stimulation, scutellarin treatment drastically reduced pro-IL-1 $\beta$ expression while also moderately decreasing the expression of pro-caspase-1 but not NLRP3 and ASC in the cell lysates (Figures $\mathbf{2 A , E - H}$ ); the reason underlying this difference is unclear and needs further investigation. Together, these results indicated that scutellarin inhibited both ATP- and nigericin-induced NLRP3 inflammasome activation in murine macrophages.

\section{Scutellarin Inhibited Pyroptosis in Macrophages upon NLRP3 Activation}

As NLRP3 inflammasome activation results in caspase-1 activation leading to pyroptosis and release of danger signals including HMGB1, we next explored whether scutellarin had any effect on ATP- or nigericin-induced pyroptosis in LPSprimed BMDMs. The pyroptotic cell death was revealed by propidium iodide (PI) staining. As shown in Figures 3A,B, ATP treatment induced pyroptosis in $\sim 35 \%$ of the cells. Notably, when compared with ATP alone group, scutellarin treatment before ATP stimulation markedly reduced pyroptosis rates in a dose-dependent manner. To further corroborate this, scutellarin also dose-dependently reduced the release of HMGB1 from the macrophages into the culture supernatants (Figures 3C,D). Without ATP triggering, however, scutellarin alone induced neither pyroptosis nor HMGB1 release in these cells. Similarly, scutellarin dose-dependently reduced nigericin-induced pyroptosis in LPS-primed macrophages (Figures 4A,B), and HMGB1 release into the culture supernatants (Figures $4 \mathrm{C}, \mathrm{D}$ ). Together these results showed that scutellarin treatment inhibited NLRP3 inflammasome activation and pyroptosis in LPS-primed murine macrophages upon ATP or nigericin stimulation.

\section{NLRP3-Mediated Formation of ASC Specks in Macrophages Was Blocked by Scutellarin}

Upon treatment with NLRP3 activators, the adaptor ASC is recruited by NLRP3 to form one large speck in each macrophage, which can be revealed by immunofluorescence microscopy. The results showed that ASC was diffusely distributed in LPS or LPS with scutellarin-treated cells. Upon ATP or nigericin treatment, ASC specks were formed in $\sim 45 \%$ or $\sim 40 \%$ of the cells, whereas scutellarin pre-treatment before ATP or nigericin stimulation markedly reduced the percentages of cells containing ASC specks to $10 \%$ or $3 \%$, respectively (Figures $5 \mathbf{A}, \mathbf{B}, \mathbf{6 A}, \mathbf{B}$ ). Consistent with previous reports (Wang et al., 2013; Granata et al., 2015), ASC specks were highly co-localized with NLRP3 in the cytoplasm, indicative of the recruitment of ASC by NLRP3 (Figures 5A, 6A). These results indicated that scutellarin inhibited NLRP3 activation by blocking ASC speck formation.

Apart from assaying ASC speck formation, we used chemical cross-linking to detect ASC oligomerization, which also reflects the activation of NLRP3 inflammasome (He et al., 2014; Coll et al., 2015). Different ASC multimers (dimers, trimers and higher oligomers) together with monomers were detected in ATP- or nigericin-treated macrophages, but were not detectable in both vehicle- and scutellarin-treated cells without ATP or nigericin. Similar to ASC speck assay, ATP- or nigericin-induced formation of ASC multimers was markedly attenuated by scutellarin pre-treatment (Figures 5C, 6C). Therefore, these data further confirmed that scutellarin inhibited NLRP3 inflammasome activation by blocking ASC recruitment to form ASC specks in macrophages.

\section{Blocking PKA Signaling Reversed Scutellarin-Mediated Suppression of ASC Specks}

The above-mentioned data indicated that scutellarin suppressed NLRP3 inflammasome activation by blocking ASC speck formation. As PKA signaling has been recently demonstrated to phosphorylate and suppress NLRP3 activation (Yan et al., 2015; Guo et al., 2016; Mortimer et al., 2016), we next explored whether PKA signaling was involved in scutellarin-mediated suppression of ASC speck formation upon NLRP3 inflammasome activation. We thus sought to determine whether scutellarin could enhance phosphorylation of NLRP3 on PKA-specific sites. A specific antibody against $\mathrm{p}$-Ser/Thr PKA substrate was used to analyze NLRP3 phosphorylation. The results showed that scutellarin treatment significantly enhanced NLRP3 phosphorylation on PKA-specific sites in LPS-primed macrophages. Further, scutellarin-mediated augmentation of NLRP3 phosphorylation was completely abrogated by PKA inhibitor H89 (Figures 7A,B).

Next, we detected ASC speck formation in the presence or absence of PKA inhibitor H89 by immunofluorescence microscopy. Notably, blocking PKA signaling by H89 not only significantly enhanced ATP-induced ASC speck formation, but 


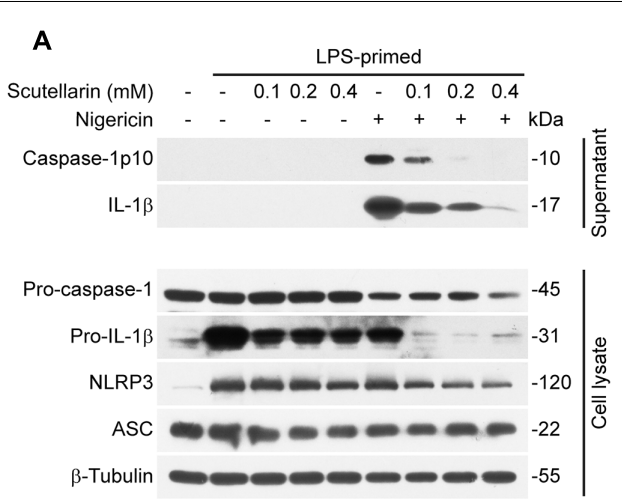

C

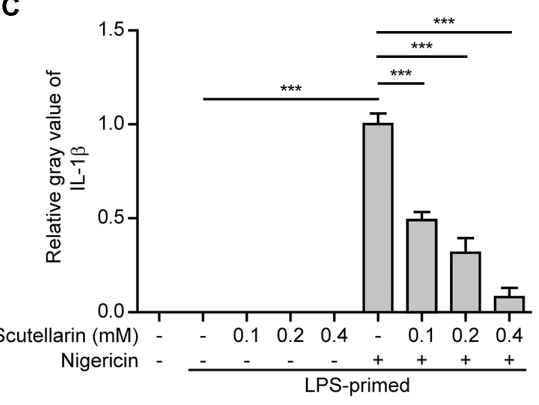

E

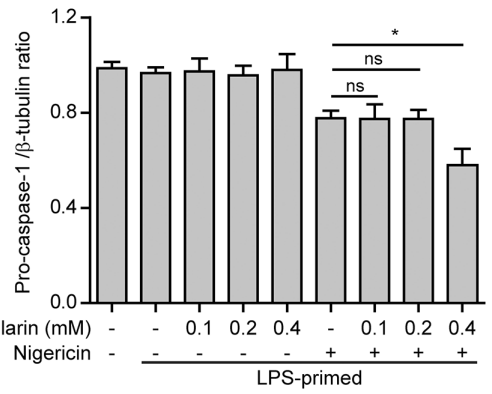

G

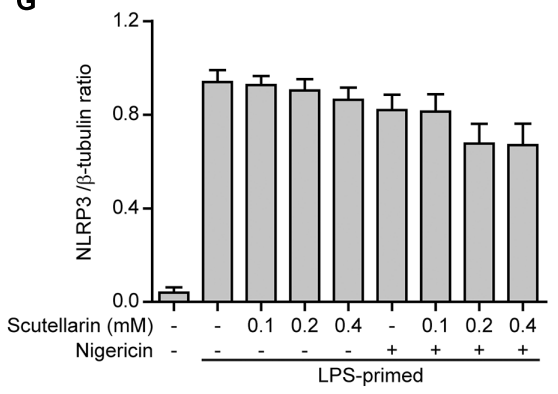

B

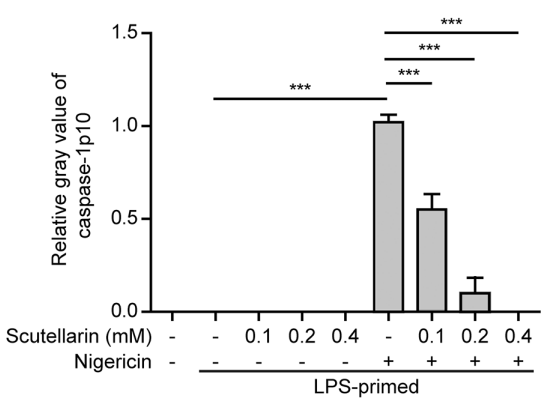

D

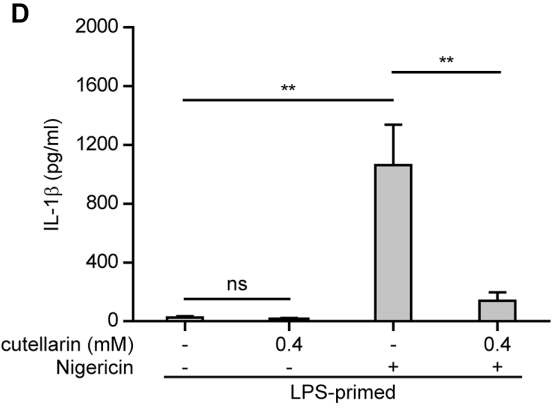

$\mathbf{F}$

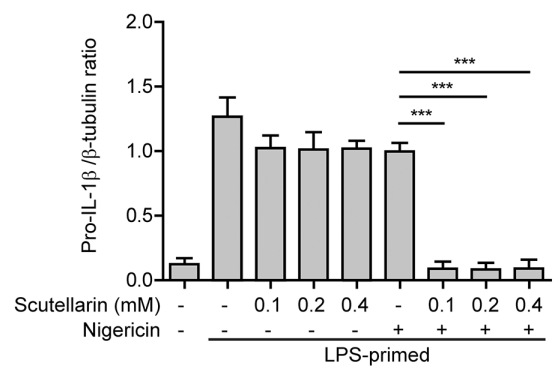

H

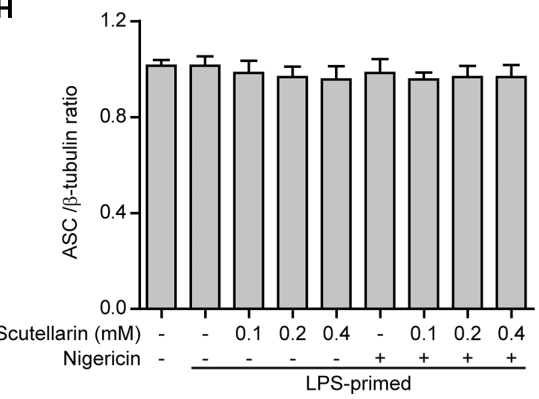

FIGURE 2 | Scutellarin inhibited nigericin-induced caspase-1 activation and mature IL-1 $\beta$ secretion. Bone marrow-derived macrophages (BMDMs) were first primed with LPS $(500 \mathrm{ng} / \mathrm{ml})$ for $4 \mathrm{~h}$, and then pre-treated with indicated doses of scutellarin for $1 \mathrm{~h}$, followed by incubation with nigericin (10 $\mu \mathrm{M}$ ) for $1 \mathrm{~h}$ in the absence of LPS. (A) Western blot analysis of indicated proteins in culture supernatants and cell lysates. $\beta$-Tubulin was recruited as a loading control for cell lysates. See Supplementary Material for source blots. (B,C) Histograms showing the relative gray values of capase-1p10 (B) or mature IL-1 $\beta$ (C) in $\mathbf{( A )}(n=3)$. The gray value of capase-1p10 or IL-1 $\beta$ bands in nigericin group was set to 1.0 and those of the other groups were relative to the ATP group. (D) Cells were treated as in (A). Cytometric bead array (CBA) was used to measure IL-1 $\beta$ levels in the culture supernatants. Data are shown as mean $\pm \mathrm{SD}(n=3)$. (E-H) Histograms showing the ratios of gray values of indicated proteins relative to $\beta$-tubulin in $(\mathbf{A})(n=3)$. ${ }^{*} P<0.05 ;{ }^{* *} P<0.01$; ${ }^{* * *} P<0.001$; ns, not significant.

also completely abrogated scutellarin-mediated suppression of ATP-induced ASC speck formation in macrophages (Figures 7C,D). H89 alone did not induce ASC speck formation. These data indicated that scutellarin inhibited ASC speck formation upon NLRP3 inflammasome activation by regulating PKA signaling. Together, these results indicated 

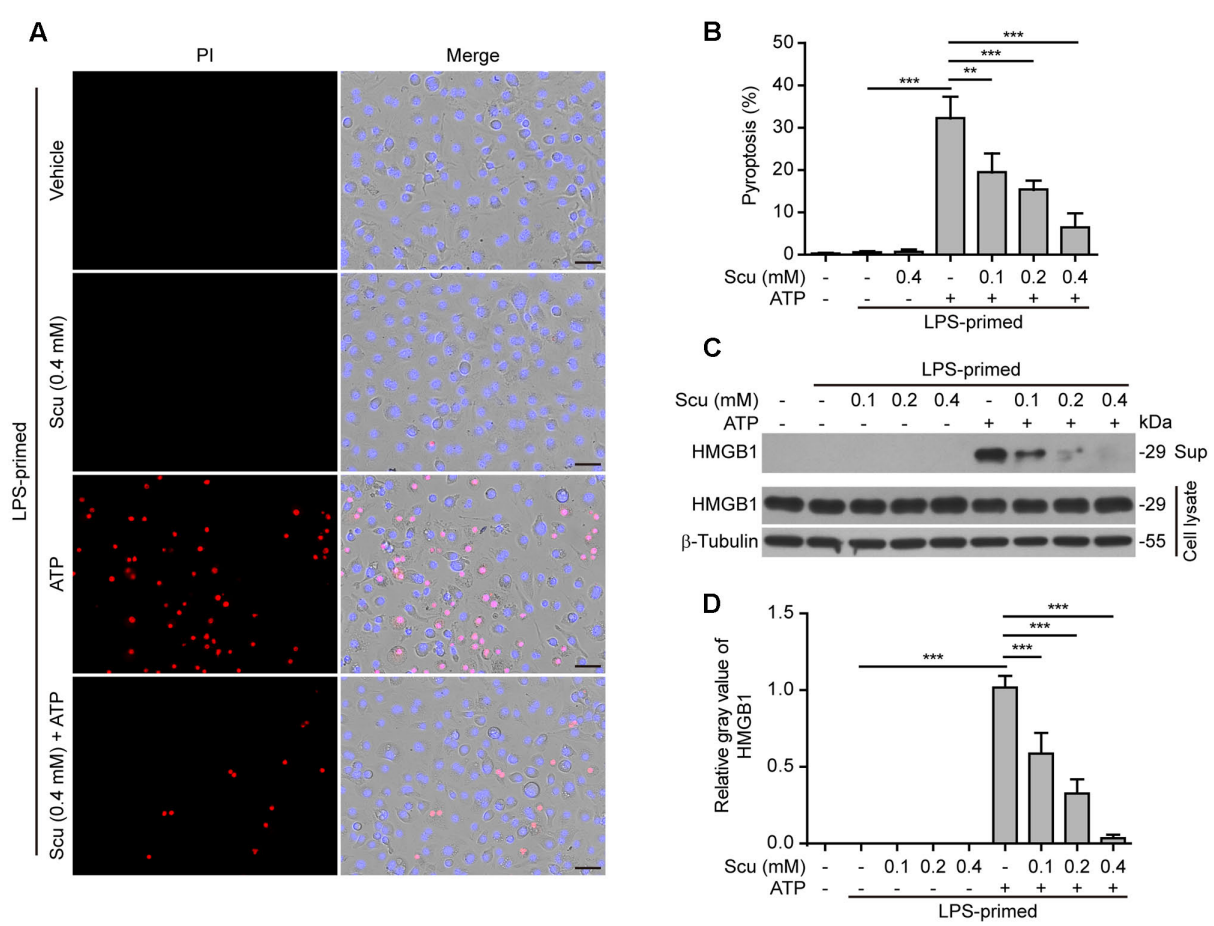

FIGURE 3 | Scutellarin inhibited ATP-induced pyroptosis in macrophages. Bone marrow-derived macrophages (BMDMs) were treated as in Figure 1A. (A) Cell death was measured by staining with propidium iodide (PI) (red, staining dead cells) and Hoechst 33342 (blue, staining all cells) for 10 min. All images were captured by fluorescence microscopy, and the merged images show PI and Hoechst 33342 fluorescence with bright-field images. One set of representative images of three independent experiments is shown. Scale bars, $50 \mu \mathrm{m}$. (B) PI-positive cells in 5 randomly chosen fields each containing $\sim 100$ cells were quantified. The percentage of pyroptosis is defined as the ratio of PI-positive relative to all (revealed by Hoechst) cells. Data are shown as mean $\pm \mathrm{SD}(n=5)$. (C) Cells were treated as in (A). Western blotting was used to assess the expression levels of indicated proteins in the cell lysates and culture supernatants (Sup), respectively. $\beta$-Tubulin was adopted as a loading control for cell lysates. See Supplementary Material for source blots. (D) Histograms showing the relative gray values of HMGB1 in (C). The gray value of HMGB1 band in ATP group was set to 1.0 and those of the other groups were relative to the ATP group. Data are shown as mean \pm SD $(n=3)$. ${ }^{* *} P<0.01 ;{ }^{* * *} P<0.001$; Scu, scutellarin.

that scutellarin-induced inhibition of ASC speck formation upon NLRP3 inflammasome activation was mediated by PKA signaling.

\section{Blockade of PKA Signaling Abrogated Scutellarin-Mediated Suppression of NLRP3 Inflammasome Activation and Pyroptosis}

We next investigated the role of PKA signaling induced by scutellarin on NLRP3 activation and pyroptosis. BMDMs were primed with LPS, pre-treated with the selective PKA inhibitor H89, and then co-incubated with scutellarin followed by ATP treatment to induce NLRP3 inflammasome activation and pyroptosis. The results showed that ATP-induced pyroptosis was markedly enhanced by H89 treatment. Of note, scutellarin-mediated suppression of ATP-induced pyroptosis was completely abrogated by H89 treatment (Figures 8A,B). These results suggested that scutellarin-induced inhibition of NLRP3 activation was dependent on PKA signaling.

Further, we sought to explore whether adenyl cyclase (the upstream component of PKA signaling) also blocked scutellarin's inhibitory action on NLRP3 inflammasome activation. The specific adenyl cyclase inhibitor MDL12330A was used to block cAMP production. Similar to H89 treatment, MDL12330A not only increased ATP-induced pyroptosis but also significantly reversed scutellarin-mediated suppression of ATP-induced pyroptosis (Figures 9A,B). Consistent with this, scutellarin-mediated suppression of caspase-1p10 and mature IL-1 $\beta$ (17 $\mathrm{kDa})$ release into the culture supernatants of BMDMs were also markedly reversed by MDL12330A treatment (Figures 9C-E). Together, these results indicated that scutellarin inhibited NLRP3 inflammasome activation and pyroptosis by augmenting PKA signaling in murine macrophages.

\section{Scutellarin Administration Protected Mice against Bacterial Sepsis}

As ATP-induced NLRP3 activation and pyroptosis plays a critical role in sepsis of bacterial infection (Wegiel et al., 2014), we finally explored whether scutellarin could attenuate bacterial sepsis in a mouse model of intraperitoneal infection. As shown in Figure 10A, mice infected with viable E. coli $\left(2.5 \times 10^{9}\right.$ $\mathrm{CFU} /$ mouse) died within $24 \mathrm{~h}$, whereas oral administration of scutellarin significantly prolonged mouse survival of bacterial sepsis. When administered with low-dose $(100 \mathrm{mg} / \mathrm{kg})$ and high-dose $(200 \mathrm{mg} / \mathrm{kg})$ scutellarin, 20 and $40 \%$ of mice 

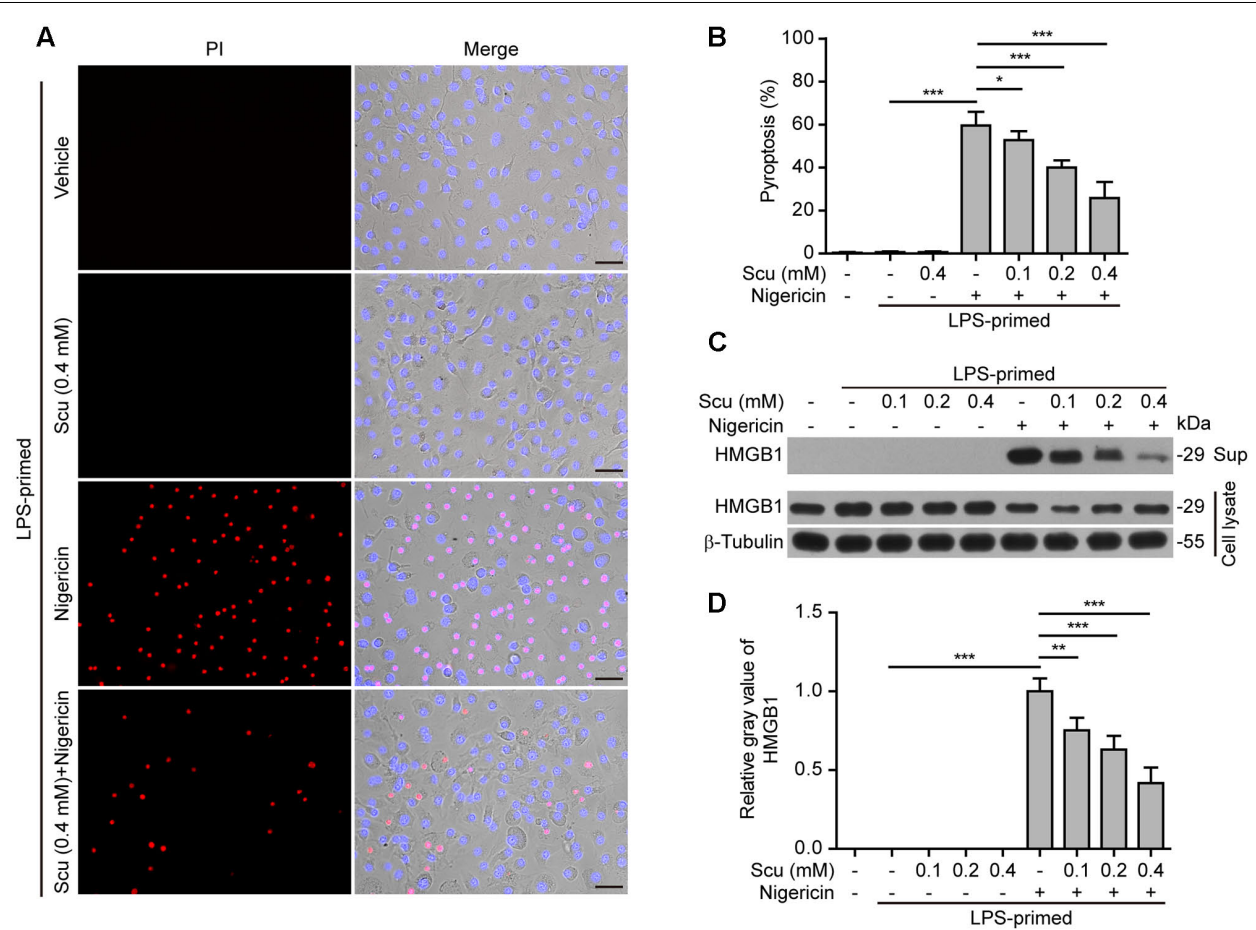

FIGURE 4 | Scutellarin inhibited nigericin-induced pyroptosis in macrophages. Bone marrow-derived macrophages (BMDMs) were treated as in Figure 2A. (A) Cell death was measured by staining with propidium iodide (PI) (red, staining dead cells) and Hoechst 33342 (blue, staining all cells) for 10 min. Fluorescent images were captured by fluorescence microscopy, and merged with bright-field images. One set of representative images of three independent experiments is shown. Scale bars, $50 \mu \mathrm{m}$. (B) PI-positive cells in 5 randomly chosen fields each containing $\sim 100$ cells were quantified. The percentage of pyroptosis is defined as the ratio of PI-positive relative to all (revealed by Hoechst) cells. Data are shown as mean $\pm \operatorname{SD}(n=5)$. (C) Cells were treated as in (A). Western blotting was used to assess the expression levels of indicated proteins in the cell lysates and culture supernatants (Sup), respectively. $\beta$-Tubulin was used as a loading control for cell lysates. See Supplementary Material for source blots. (D) Histograms showing the relative gray values of HMGB1 in (C). The gray value of HMGB1 band in nigericin group was set to 1.0 with those of the other groups being calculated relative to the nigericin group. Data are shown as mean $\pm \mathrm{SD}(n=3)$. ${ }^{*}<0.05$; ${ }^{* *} P<0.01$;

$* * * P<0.001 ;$ Scu, scutellarin.

had survived the period of observation (120 h), respectively (Figure 10A). In addition, scutellarin treatment significantly reduced serum IL-1 $\beta$ levels and attenuated infiltration of inflammatory cells in the liver of mice infected with E. coli in comparison to vehicle (Figures 10B,C), indicating that scutellarin inhibited systemic inflammation in the mice. But there was no obvious lesion in the jejunum and colon tissues with or without scutellarin administration (Supplementary Figure S1). Scutellarin had no influences on IL-1 $\beta$ levels in uninfected (control) mice (Figure 10B). The bacterial loads were also unchanged by scutellarin administration (Supplementary Figure S2). As IL-1 $\beta$ secretion in vivo upon Gram-negative bacterial infection has been shown to be NLRP3 dependent (He et al., 2013), these results suggested that scutellarin protected mice against bacterial sepsis probably by suppressing NLRP3 inflammasome activation upon microbial infection.

\section{DISCUSSION}

Scutellarin has been shown to have potent anti-inflammatory activities (Tan et al., 2010; Wang et al., 2011; Chen et al., 2013; Yuan et al., 2014; Niu et al., 2015; Wang W. et al., 2016; Zhao et al., 2016). But it was unclear whether this agent has any potential effects on NLRP3 inflammasome activation, which has been implicated in many inflammatory conditions ranging from metabolic disorders to bacterial sepsis. In the current study, we found that scutellarin robustly inhibited ATP- or nigericininduced NLRP3 inflammasome activation and pyroptosis in murine macrophages, thus unraveling a previously unappreciated action mechanism for scutellarin in preventing inflammatory responses in tissue injury and bacterial infections.

NLRP3 can be activated by a wide range of activators including ATP and nigericin (Mariathasan et al., 2006). It is thought that ATP acts on its receptor P2X7 adrenergic receptor, leading to the opening of potassium ion channel and the efflux of $\mathrm{K}^{+}$. The efflux of $\mathrm{K}^{+}$in turn triggers the assembly of NLRP3 inflammasome; being different from ATP, nigericin is a potassium ion carrier and can insert into the plasma membrane to form potassium channels which cause the efflux of $\mathrm{K}^{+}$, thus activating the NLRP3 inflammasome (Munoz-Planillo et al., 2013; He et al., 2016). We found that scutellarin inhibited NLRP3 inflammasome activation by both ATP and nigericin, suggesting that its action may on the downstream components of P2X7 receptor. Supporting this idea, our study showed that scutellarin markedly suppressed ASC speck formation and oligomerization upon NLRP3 activation 

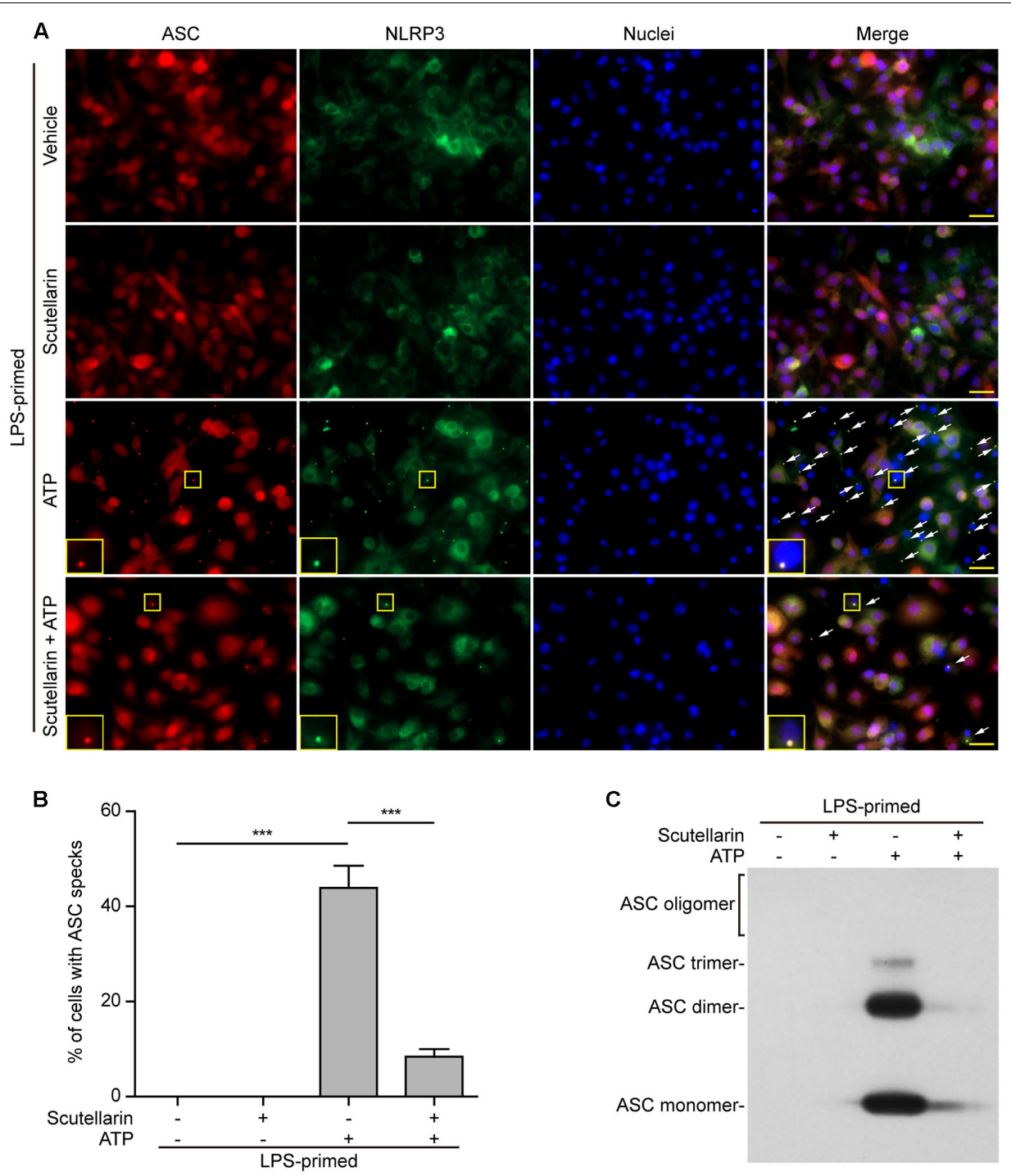

FIGURE 5 | Scutellarin blocked ATP-induced ASC speck formation and oligomerization in macrophages. Bone marrow-derived macrophages (BMDMs) were first primed with LPS $(500 \mathrm{ng} / \mathrm{ml})$ for $4 \mathrm{~h}$, and then the cells pre-treated with scutellarin $(400 \mu \mathrm{M})$ for $1 \mathrm{~h}$, followed by incubation with ATP (3 mM) for 30 min without LPS (A) Representative immunofluorescence images showing ASC (red) and NLRP3 (green) subcellular distribution. Nuclei (blue) were revealed by Hoechst 33342 . The merged images are presented to show the co-localization of ASC with NLRP3. White arrows indicate ASC specks and the enlarged inset showing cells with an ASC speck. Scale bars, $20 \mu \mathrm{m}$. (B) Percentages of cells with an ASC speck relative to total cells from 5 random fields each containing $\sim 50$ cells. Data are shown as mean $\pm \mathrm{SD}(n=3) .{ }^{* * *} P<0.001$. (C) Western blot analysis of disuccinimidyl suberate-cross-linked pellets with anti-ASC antibody, showing ASC oligomerization in each sample.

by ATP or nigericin, suggesting their interference of NLRP3's capacity in recruiting ASC to form inflammasomes.

One major concern is how scutellarin suppressed NLRP3's capacity to assemble inflammasomes upon ATP or nigericin stimulation. Recent studies have demonstrated that NLRP3 activation has been negatively regulated by PKA signaling (Kim et al., 2017), which can be augmented by small molecules, such as bile acids (Guo et al., 2016), prostaglandin E2 (Mortimer et al., 2016), and dopamine (Yan et al., 2015), through their respective receptors; Ser/Thr phosphorylation of NLRP3 on PKA-specific sites prevents it from assembling inflammasomes. In light of the fact that scutellarin can increase cAMP levels (Tian et al., 2016) (and therefore increases the PKA activity), we proposed that scutellarin might block NLRP3 activation by enhancing PKA signaling. In support of this notion, selective PKA inhibitor H89 completely abrogated the inhibitory effects of scutellarin on pyroptosis in macrophages. H89 also augmented ATP-induced formation of ASC specks and entirely reversed scutellarin-mediated suppression of ASC specks. Accompanying this, scutellarin-induced phosphorylation of NLRP3 on PKA-specific sites was also blocked by H89 pretreatment. Besides, MDL12330A, an inhibitor of adenyl cyclase (AC) upstream of PKA signaling, also drastically abrogated scutellarin's inhibitory effects on NLRP3 activation 


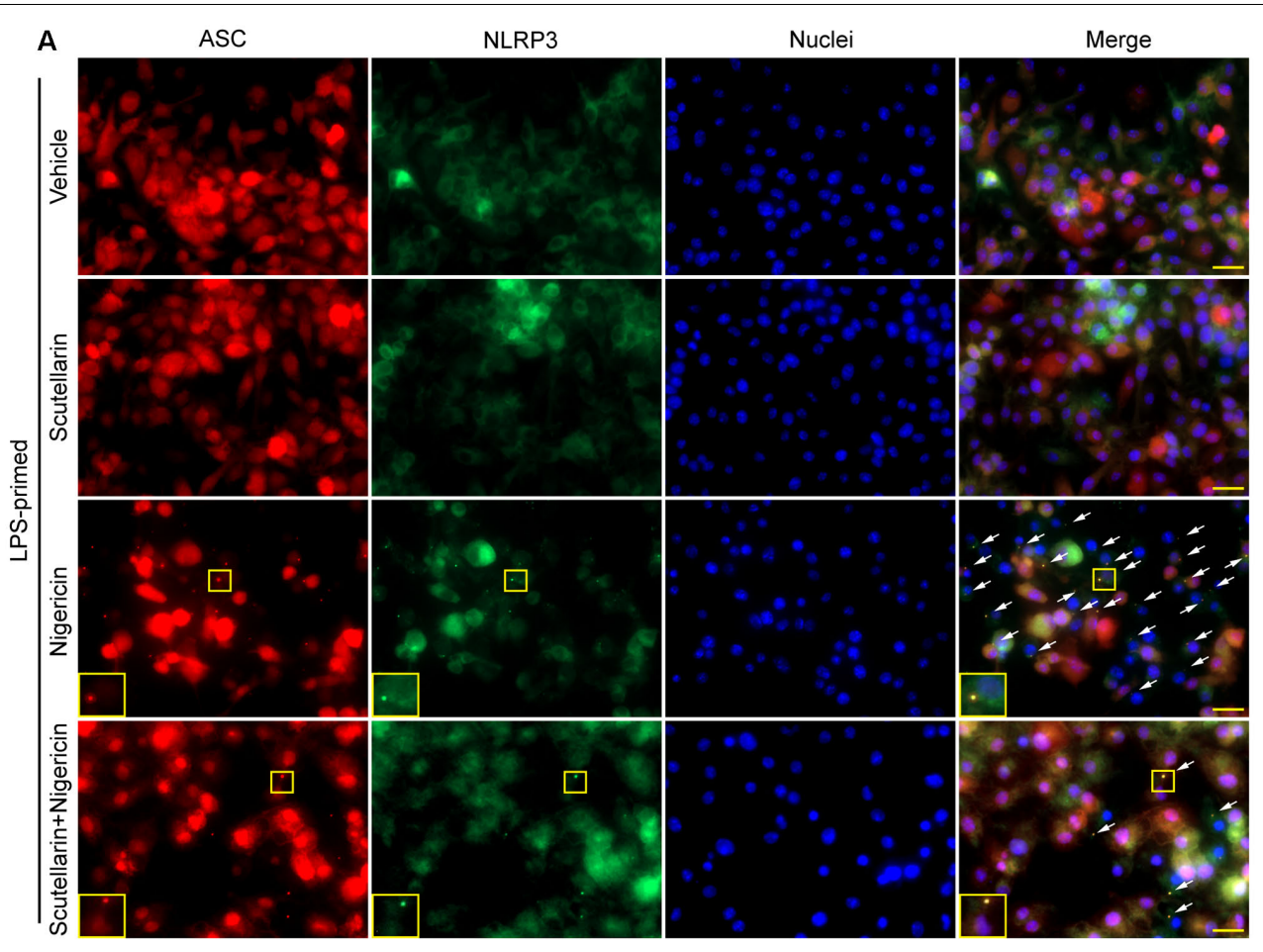

B

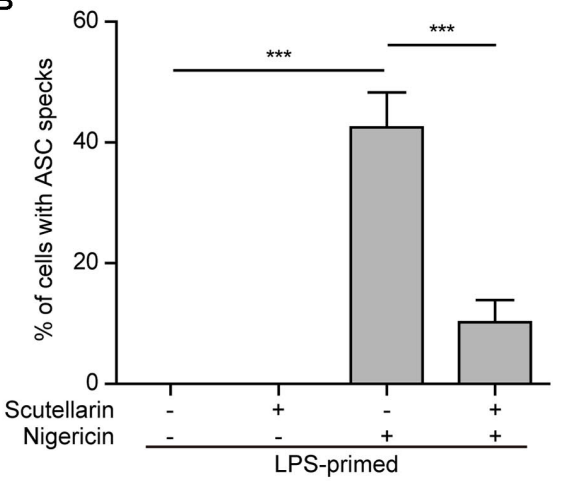

C

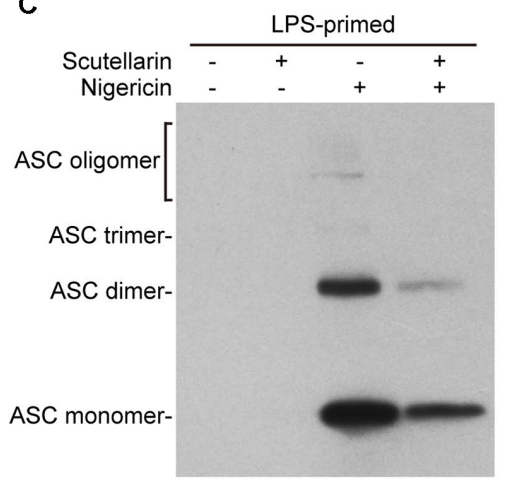

FIGURE 6 | Scutellarin blocked nigericin-induced ASC speck formation and oligomerization in macrophages. Bone marrow-derived macrophages (BMDMs) were first primed with LPS $(500 \mathrm{ng} / \mathrm{ml})$ for $4 \mathrm{~h}$, and then pre-treated with scutellarin $(400 \mu \mathrm{M})$ for $1 \mathrm{~h}$, followed by incubation with nigericin (10 $\mu \mathrm{M})$ for $1 \mathrm{~h}$ without LPS. (A) Representative immunofluorescence images showing ASC (red) and NLRP3 (green) subcellular distribution. Nuclei (blue) were revealed by Hoechst 33342 . The merged images are presented to show the co-localization of ASC with NLRP3. White arrows indicate ASC specks and the insets show cells with an ASC speck. Scale bars, $20 \mu \mathrm{m}$. (B) Quantification of ASC speck formation by the number of cells with ASC specks relative to the total number of cells from 5 random fields each containing $\sim 50$ cells. Data are shown as mean $\pm \operatorname{SD}(n=3)$. ${ }^{* * *} P<0.001$. (C) Western blotting of disuccinimidyl suberate-cross-linked pellets with anti-ASC antibody, showing ASC oligomerization in each sample.

and pyroptosis. Altogether, these data indicated that scutellarin inhibited NLRP3 inflammasome activation and pyroptosis in macrophages by augmenting PKA signaling.

The formation of NLRP3 inflammasome provides a platform for the activation of caspase- 1 , which in turn converts pro-IL-1 $\beta$ into mature IL-1 $\beta$. The release of mature IL-1 $\beta$ has been shown to be dependent on gasdermin D-mediated pyroptosis (He et al., 2015; Shi et al., 2015). Active caspase-1 leads to pyroptosis by cleaving gasdermin $\mathrm{D}$ to generate $\mathrm{N}$-terminal product (gasdermin D-NT), which forms pores on the plasma membrane culminating in pyroptotic cell death (Chen et al., 2016; Ding et al., 2016; Liu et al., 2016; Sborgi et al., 2016). Consistent with these studies, our results showed that scutellarin not only inhibited caspase-1 activation and pyroptosis but also blocked mature IL-1 $\beta$ secretion into culture supernatants. Accompanying the suppression of mature IL-1 $\beta$ secretion, the release of other inflammatory intracellular components including HMGB1 had also been reduced. As HMGB1 is a critical damage-associated molecular pattern (DAMP) that is released into extracellular milieu during bacterial infections or cell 

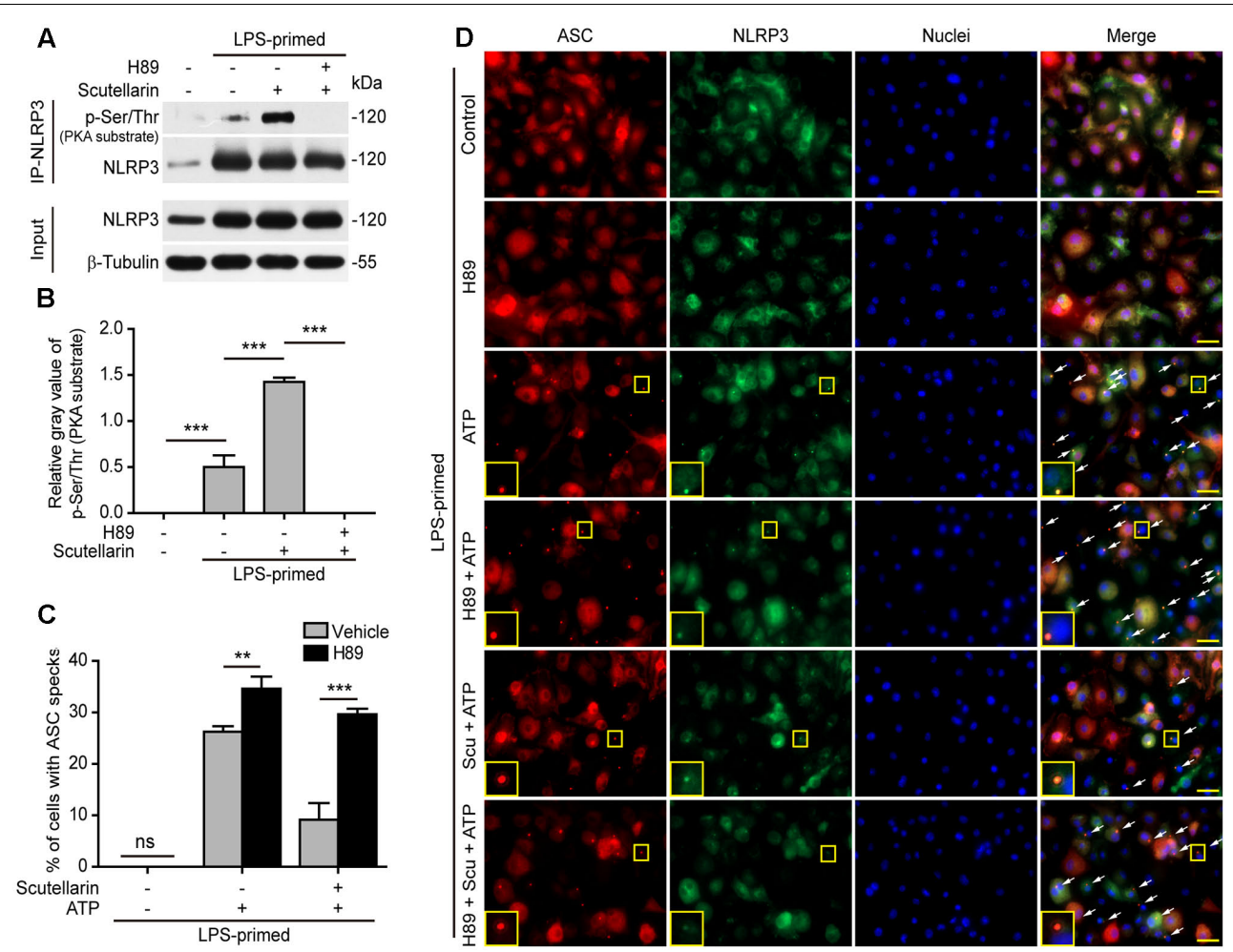

FIGURE 7 | Scutellarin inhibited NLRP3 inflammasome activation via PKA signaling. (A) LPS-primed BMDMs were incubated with the selective PKA inhibitor H89 $(20 \mu \mathrm{M})$ for $30 \mathrm{~min}$, and then treated with scutellarin $(400 \mu \mathrm{M})$ for $1 \mathrm{~h}$. NLRP3 was immunoprecipitated from the cell lysates and analyzed for its Ser/Thr phosphorylation on PKA-specific sites (p-Ser/Thr). See Supplementary Material for source blots. (B) The gray values of NLRP3 phosphorylation on PKA-specific sites (p-Ser/Thr) relative to total NLRP3 in (A). (C) BMDMs were treated as in Figure 5. Immunofluorescence images were obtained to show ASC (red) and NLRP3 (green) subcellular distribution. Nuclei (blue) were revealed by Hoechst 33342. White arrows indicate ASC specks and the insets show enlarged cells with an ASC speck. Scale bars, $20 \mu \mathrm{m}$. (D) Percentages of cell containing an ASC speck relative to the total cells from 5 random fields each containing $\sim 50$ cells. ${ }^{* *} P<0.01$; ***P $<0.001$; ns, not significant; Scu, scutellarin.

damages triggering multiple inflammatory signaling (Lotze and Tracey, 2005; Yanai et al., 2012), the anti-inflammatory activities of scutellarin may be partly mediated by suppressing HMGB1 release during NLRP3-dependent pyroptosis. Thus, combined suppression of mature IL- $1 \beta$ release and DAMPs such as HMGB1 may account for the potent anti-inflammatory activities of scutellarin, highlighting its potency against NLRP3-mediated inflammation.

The activation of NLRP3 inflammasome has been suggested to play important roles in sepsis (Gong et al., 2015; Kim et al., 2016; Long et al., 2016; Hao et al., 2017; Lee et al., 2017), which is currently defined as life threatening organ dysfunction caused by a dysregulated host response to infection (Singer et al., 2016). In the early stages of sepsis, large amounts of proinflammatory cytokines including IL-1 $\beta$, known as 'cytokine storm; are produced leading to multiple organ injury and failure (van der Poll et al., 2017). Therefore, in the initial phase of sepsis, the anti-inflammatory therapy is effective and blocking the activity of IL- $1 \beta$ with biologic inhibitors has been used to prevent sepsis but showed limited effectiveness (Marshall, 2014). Recent studies proposed that blockade of NLRP3 activation, thus blocking the secretion of IL-1 $\beta$ and other inflammatory components, might have advantages over the use of biologic inhibitors of IL-1 $\beta$. For example, small molecules, such as glyburide (Lamkanfi et al., 2009), MCC950 (Coll et al., 2015), and ketone metabolite $\beta$-hydroxybutyrate (Youm et al., 2015), have been shown to be inhibitors of NLRP3 inflammasome, thus being effective in treating NLRP3-related diseases. Consistent with these studies, we showed that oral administration of scutellarin significantly improved the survival of mice in bacterial sepsis. In line with the in vitro data, scutellarin administration also markedly decreased serum IL-1 $\beta$ levels and reduced infiltration of inflammatory cells in the liver. However, the bacterial burden in the peritoneal cavity was unchanged by scutellarin suggesting that it had no antibacterial activity, which is consistent with previous study showing no bactericidal activity for scutellarin (Lucarini et al., 2015). Thus, the improved survival of mice with bacterial sepsis was most likely due to attenuated systemic inflammation, as revealed by decreased serum IL-1 $\beta$ levels and reduced infiltration of inflammatory cells in the liver. These results suggest that scutellarin may be a potential therapeutic agent for the treatment of bacterial sepsis.

Previous studies had shown that $50 \mathrm{mg} / \mathrm{kg}$ and $100 \mathrm{mg} / \mathrm{kg}$ body weight of scutellarin (either intraperitoneally or orally administered) significantly attenuates inflammatory responses 

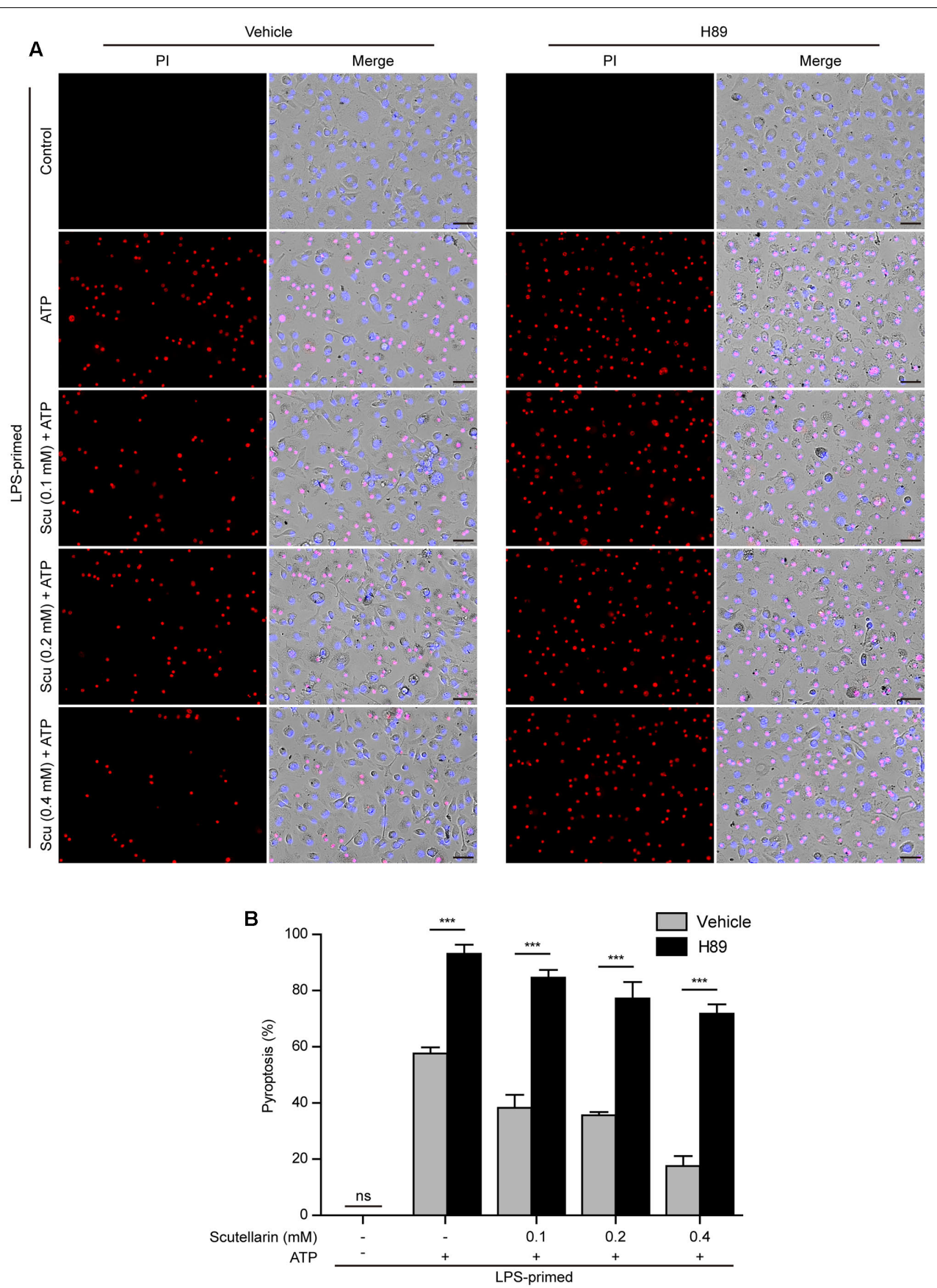

FIGURE 8 | Scutellarin-mediated suppression of NLRP3 inflammasome activation was abrogated by PKA inhibitor. LPS-primed bone marrow-derived macrophages (BMDMs) were pre-treated with the selective PKA inhibitor H89 (20 $\mu \mathrm{M})$ for $30 \mathrm{~min}$, and then incubation with indicated doses of scutellarin for $1 \mathrm{~h}$, followed by stimulation with ATP ( $3 \mathrm{mM}$ ) for $30 \mathrm{~min}$. (A) Cell death was assayed by propidium iodide (PI) (red) and Hoechst 33342 (blue) co-staining for 10 min. The images were captured by fluorescence microscopy. One set of representative images of three independent experiments is shown. Scale bars, $50 \mu \mathrm{m}$. (B) PI-positive cells in 5 randomly chosen fields each containing $\sim 100$ cells were quantified. The percentage of pyroptosis is defined as the ratio of PI-positive relative to all (revealed by Hoechst) cells. Data are shown as mean $\pm \mathrm{SD}(n=5)$. ${ }^{* * * P}<0.001$; ns, not significant; Scu, scutellarin.

in a rat model of brain ischemia injury (Yuan et al., 2014; Wang W. et al., 2016). Another toxicity study indicated that oral administration of scutellarin at $500 \mathrm{mg} / \mathrm{kg}$ body weight daily for 30 days did not result in death or significant changes in hematology, blood chemistries or urinalysis, indicating that scutellarin is minimally toxic or non-toxic in rodents (Li et al., 2011). Based on these previous studies, we used $100 \mathrm{mg} / \mathrm{kg}$ and $200 \mathrm{mg} / \mathrm{kg}$ body weight of scutellarin for animal experiments. Instead of intraperitoneal injection, scutellarin was administered via oral gavage in order to avoid direct interference with bacteria 

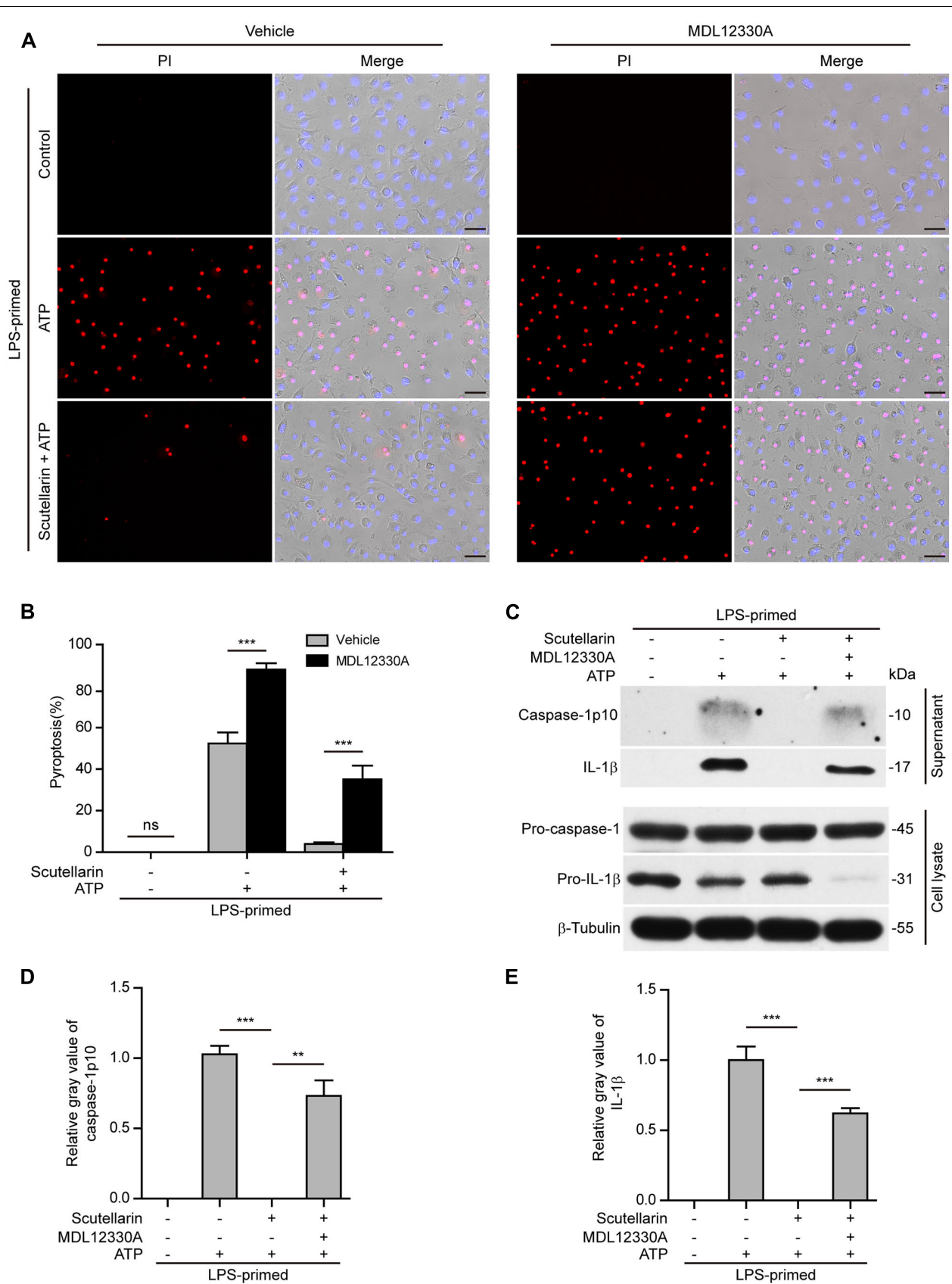

FIGURE 9 | Scutellarin-mediated suppression of NLRP3 inflammasome activation was blocked by adenyl cyclase inhibitor. Bone marrow-derived macrophages (BMDMs) were primed with LPS (500 ng/ml) for $4 \mathrm{~h}$, and pre-treated with scutellarin (400 $\mu \mathrm{M})$ for 30 min before incubation with inhibitor MDL12330A (10 $\mu \mathrm{M})$ for $30 \mathrm{~min}$, followed by co-treatment with ATP (3 mM) for $30 \mathrm{~min}$. (A) Cell death was measured by staining with propidium iodide (PI) (red, staining dead cells) and Hoechst 33342 (blue, staining all cells) for 10 min. Fluorescent images were captured by fluorescent microscopy, and merged with bright-field images. One set of representative images of three independent experiments is shown. Scale bars, $50 \mu \mathrm{m}$. (B) PI-positive cells in 5 randomly chosen fields each containing $\sim 100$ cells were quantified. The percentage of pyroptosis is defined as the ratio of PI-positive relative to all (revealed by Hoechst) cells. Data are shown as mean $\pm \mathrm{SD}(n=5)$. (C) Western blot analysis was used to detect the expression and secretion of caspase- 1 and IL- $1 \beta$ in the cell lysates and culture supernatants, respectively. $\beta$-Tubulin was used as a loading control for cell lysates. See Supplementary Material for source blots. (D,E) Histograms showing the relative gray values of capase-1p10 (D) or $\mathrm{IL}-1 \beta \mathbf{~ ( E ) ~ i n ~ ( C ) . ~ T h e ~ g r a y ~ v a l u e ~ o f ~ c a p a s e - 1 p 1 0 ~ o r ~ I L - 1 \beta ~ b a n d s ~ i n ~ A T P ~ g r o u p ~ w a s ~ s e t ~ t o ~} 1.0$ and those of the other groups were calculated relative to the ATP group. Data are shown as mean $\pm \mathrm{SD}(n=3) .{ }^{* *} P<0.01$; ${ }^{* *} P<0.001$; ns, not significant; Scu, scutellarin.

in the peritoneal cavity. Our results showed that these two doses of scutellarin significantly reduced systemic inflammation of bacterial infected mice and increased their survival rates.
These data indicate that oral administration of scutellarin may be an effective route conferring protective effects against systemic inflammation during bacterial infections. 

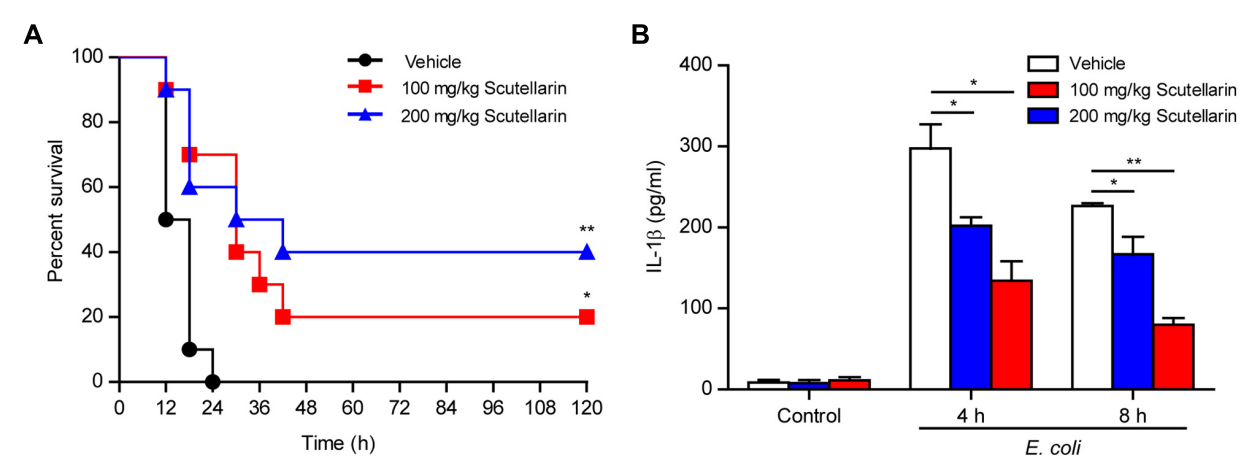

C

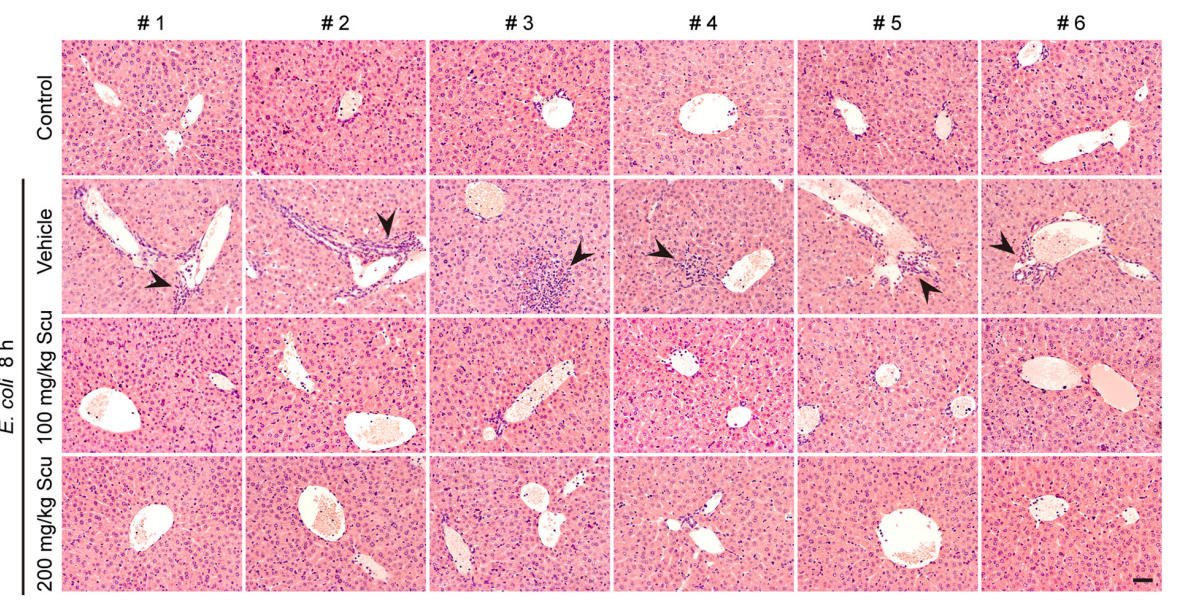

FIGURE 10 | Scutellarin administration improved the survival of mice with bacterial sepsis. C57BL/6 mice were administered once intragastrically (i.g.) with scutellarin (100 or $200 \mathrm{mg} / \mathrm{kg}$ body weight) or vehicle (2\% Tween 80 in PBS) $3 \mathrm{~h}$ prior to bacterial infection intraperitoneally (i.p.) with viable Escherichia coli $\left(2.5 \times 10^{9} \mathrm{CFU} /\right.$ mouse). Mice were administered (i.g.) with scutellarin or vehicle once again at $1 \mathrm{~h}$ after the bacterial injection. Mouse survival was monitored every $6 \mathrm{~h}$ for 5 consecutive days. Kaplan-Meier survival curves were used to analyze the data (10 mice per group). The significance was evaluated by the log-rank (Mantel-Cox) test. (B) Mice were treated as in (A) except that mice were injected (i.p.) with viable $E$. coli $\left(2.0 \times 10^{9} \mathrm{CFU} / \mathrm{mouse}\right)$. The serum levels of IL- $1 \beta$ at 4 and $8 \mathrm{~h}$ post bacterial infection were measured by CBA assay (6 mice per group). Data are shown as mean $\pm \mathrm{SD}(n=6) .{ }^{*} P<0.05 ;{ }^{* *} P<0.01$. (C) Hematoxylin and eosin staining of the liver of mice infected with live $E$. coli for $8 \mathrm{~h}$. Representative images from each mouse were presented and arrowheads indicated infiltrated inflammatory cells. The numbers on the top indicate mouse number. Scale bar, $50 \mu \mathrm{m}$; Scu, scutellarin.

\section{CONCLUSION}

We demonstrated that scutellarin inhibited NLRP3 inflammasome activation and pyroptosis in macrophages through augmenting PKA signaling, unraveling a novel anti-inflammatory mechanism beyond previously identified inhibitory action on the NF- $\mathrm{B}$ and Notch pathways (Tan et al., 2010; Yuan et al., 2014, 2015; Wang W. et al., 2016; Zhao et al., 2016). Furthermore, our data also suggest that scutellarin can be used to treat bacterial sepsis probably by blocking NLRP3 activation in the early stage of bacterial sepsis. As aberrant NLRP3 activation has also been implicated in more complex diseases including type 2 diabetes, gout, atherosclerosis and neurological diseases (Guo et al., 2015), scutellarin may have potential application in the treatment of such disorders. It is still unknown how scutellarin affects the PKA signaling pathway in macrophages. Although further research is warranted to elucidate the precise mechanism underlying this process, our data highlight the potential application of scutellarin for the treatment for NLRP3-related inflammatory diseases.

\section{AUTHOR CONTRIBUTIONS}

YL, Y-YJ, C-YZ, C-GL, and L-HX performed the cellular studies. C-GL, LY, W-JB, and Q-BZ performed the flow cytometry and immunofluorescence assays. Y-YJ and C-GL analyzed the data. $\mathrm{X}-\mathrm{HH}$ and D-YO supervised the study. X-HH, D-YO, and YL wrote the paper.

\section{FUNDING}

This study was supported by the grants from the National Natural Science Foundation of China (Nos. 81773965, 81673664, and 81373423).

\section{SUPPLEMENTARY MATERIAL}

The Supplementary Material for this article can be found online at: https://www.frontiersin.org/articles/10.3389/fphar.2017. 00975/full\#supplementary-material 


\section{REFERENCES}

Broz, P., and Dixit, V. M. (2016). Inflammasomes: mechanism of assembly, regulation and signalling. Nat. Rev. Immunol. 16, 407-420. doi: 10.1038/nri. 2016.58

Chen, X., He, W. T., Hu, L., Li, J., Fang, Y., Wang, X., et al. (2016). Pyroptosis is driven by non-selective gasdermin-D pore and its morphology is different from MLKL channel-mediated necroptosis. Cell Res. 26, 1007-1020. doi: 10.1038/cr. 2016.100

Chen, X., Shi, X., Zhang, X., Lei, H., Long, S., Su, H., et al. (2013). Scutellarin attenuates hypertension-induced expression of brain Toll-like receptor 4/nuclear factor kappa B. Mediators Inflamm. 2013:432623. doi: 10.1155/2013/432623

Coll, R. C., Robertson, A. A., Chae, J. J., Higgins, S. C., Munoz-Planillo, R., Inserra, M. C., et al. (2015). A small-molecule inhibitor of the NLRP3 inflammasome for the treatment of inflammatory diseases. Nat. Med. 21, 248-255. doi: 10.1038/ nm.3806

de Torre-Minguela, C., Mesa Del Castillo, P., and Pelegrin, P. (2017). The NLRP3 and pyrin inflammasomes: implications in the pathophysiology of autoinflammatory diseases. Front. Immunol. 8:43. doi: 10.3389/fimmu.2017. 00043

Ding, J., Wang, K., Liu, W., She, Y., Sun, Q., Shi, J., et al. (2016). Pore-forming activity and structural autoinhibition of the gasdermin family. Nature 535, 111-116. doi: 10.1038/nature18590

Duewell, P., Kono, H., Rayner, K. J., Sirois, C. M., Vladimer, G., Bauernfeind, F. G., et al. (2010). NLRP3 inflammasomes are required for atherogenesis and activated by cholesterol crystals. Nature 464, 1357-1361. doi: 10.1038/ nature 08938

Gao, J., Chen, G., He, H., Liu, C., Xiong, X., Li, J., et al. (2017). Therapeutic effects of breviscapine in cardiovascular diseases: a review. Front. Pharmacol. 8:289. doi: 10.3389/fphar.2017.00289

Gao, M., Huang, W., and Liu, C. Z. (2007). Separation of scutellarin from crude extracts of Erigeron breviscapus (vant.) Hand. Mazz. by macroporous resins. J. Chromatogr. B Analyt. Technol. Biomed. Life Sci. 858, 22-26. doi: 10.1016/j. jchromb.2007.07.046

Gong, Z., Zhou, J., Li, H., Gao, Y., Xu, C., Zhao, S., et al. (2015). Curcumin suppresses NLRP3 inflammasome activation and protects against LPS-induced septic shock. Mol. Nutr. Food Res. 59, 2132-2142. doi: 10.1002/mnfr.201500316

Granata, S., Masola, V., Zoratti, E., Scupoli, M. T., Baruzzi, A., Messa, M., et al. (2015). NLRP3 inflammasome activation in dialyzed chronic kidney disease patients. PLOS ONE 10:e0122272. doi: 10.1371/journal.pone.0122272

Guo, C., Xie, S., Chi, Z., Zhang, J., Liu, Y., Zhang, L., et al. (2016). Bile acids control inflammation and metabolic disorder through inhibition of NLRP3 inflammasome. Immunity 45, 802-816. doi: 10.1016/j.immuni.2016.09.008

Guo, H., Callaway, J. B., and Ting, J. P. (2015). Inflammasomes: mechanism of action, role in disease, and therapeutics. Nat. Med. 21, 677-687. doi: 10.1038/ nm.3893

Guo, H., Hu, L. M., Wang, S. X., Wang, Y. L., Shi, F., Li, H., et al. (2011). Neuroprotective effects of scutellarin against hypoxic-ischemic-induced cerebral injury via augmentation of antioxidant defense capacity. Chin. J. Physiol. 54, 399-405. doi: 10.4077/cjp.2011.amm059

Hao, H., Cao, L., Jiang, C., Che, Y., Zhang, S., Takahashi, S., et al. (2017). Farnesoid $\mathrm{X}$ receptor regulation of the NLRP3 inflammasome underlies cholestasisassociated sepsis. Cell Metab. 25, 856.e5-867.e5. doi: 10.1016/j.cmet.2017. 03.007

He, W. T., Wan, H., Hu, L., Chen, P., Wang, X., Huang, Z., et al. (2015). Gasdermin $\mathrm{D}$ is an executor of pyroptosis and required for interleukin-1beta secretion. Cell Res. 25, 1285-1298. doi: 10.1038/cr.2015.139

He, Y., Franchi, L., and Nunez, G. (2013). TLR agonists stimulate Nlrp3-dependent IL-1beta production independently of the purinergic $\mathrm{P} 2 \mathrm{X} 7$ receptor in dendritic cells and in vivo. J. Immunol. 190, 334-339. doi: 10.4049/jimmunol.1202737

He, Y., Hara, H., and Nunez, G. (2016). Mechanism and regulation of NLRP3 inflammasome activation. Trends Biochem. Sci. 41, 1012-1021. doi: 10.1016/j. tibs.2016.09.002

He, Y., Varadarajan, S., Munoz-Planillo, R., Burberry, A., Nakamura, Y., and Nunez, G. (2014). 3,4-methylenedioxy-beta-nitrostyrene inhibits NLRP3 inflammasome activation by blocking assembly of the inflammasome. J. Biol. Chem. 289, 1142-1150. doi: 10.1074/jbc.M113.515080
Heneka, M. T., Kummer, M. P., Stutz, A., Delekate, A., Schwartz, S., VieiraSaecker, A., et al. (2013). NLRP3 is activated in Alzheimer's disease and contributes to pathology in APP/PS1 mice. Nature 493, 674-678. doi: 10.1038/ nature 11729

Ito, M., Shichita, T., Okada, M., Komine, R., Noguchi, Y., Yoshimura, A., et al. (2015). Bruton's tyrosine kinase is essential for NLRP3 inflammasome activation and contributes to ischaemic brain injury. Nat. Commun. 6:7360. doi: $10.1038 /$ ncomms 8360

Jo, E. K., Kim, J. K., Shin, D. M., and Sasakawa, C. (2016). Molecular mechanisms regulating NLRP3 inflammasome activation. Cell. Mol. Immunol. 13, 148-159. doi: $10.1038 / \mathrm{cmi} .2015 .95$

Kayagaki, N., Stowe, I. B., Lee, B. L., O’Rourke, K., Anderson, K., Warming, S., et al. (2015). Caspase-11 cleaves gasdermin D for non-canonical inflammasome signalling. Nature 526, 666-671. doi: 10.1038/nature15541

Kayagaki, N., Warming, S., Lamkanfi, M., Vande Walle, L., Louie, S., Dong, J., et al. (2011). Non-canonical inflammasome activation targets caspase-11. Nature 479, 117-121. doi: 10.1038/nature 10558

Kayagaki, N., Wong, M. T., Stowe, I. B., Ramani, S. R., Gonzalez, L. C., Akashi-Takamura, S., et al. (2013). Noncanonical inflammasome activation by intracellular LPS independent of TLR4. Science 341, 1246-1249. doi: 10.1126/ science. 1240248

Kim, J. K., Jin, H. S., Suh, H. W., and Jo, E. K. (2017). Negative regulators and their mechanisms in NLRP3 inflammasome activation and signaling. Immunol. Cell Biol. 95, 584-592. doi: 10.1038/icb.2017.23

Kim, M. J., Bae, S. H., Ryu, J. C., Kwon, Y., Oh, J. H., Kwon, J., et al. (2016). SESN2/sestrin2 suppresses sepsis by inducing mitophagy and inhibiting NLRP3 activation in macrophages. Autophagy 12, 1272-1291. doi: 10.1080/15548627. 2016.1183081

Lamkanfi, M., Mueller, J. L., Vitari, A. C., Misaghi, S., Fedorova, A., Deshayes, K., et al. (2009). Glyburide inhibits the Cryopyrin/Nalp3 inflammasome. J. Cell Biol. 187, 61-70. doi: 10.1083/jcb.200903124

Lee, S., Nakahira, K., Dalli, J., Siempos, I. I., Norris, P. C., Colas, R. A., et al. (2017). NLRP3 inflammasome deficiency protects against microbial sepsis via increased lipoxin B4 synthesis. Am. J. Respir. Crit. Care Med. 196, 713-726. doi: 10.1164/rccm.201604-0892OC

Li, C. G., Yan, L., Jing, Y. Y., Xu, L. H., Liang, Y. D., Wei, H. X., et al. (2017). Berberine augments ATP-induced inflammasome activation in macrophages by enhancing AMPK signaling. Oncotarget 8, 95-109. doi: 10.18632/oncotarget. 13921

Li, X., Wang, L., Li, Y., Bai, L., and Xue, M. (2011). Acute and subacute toxicological evaluation of scutellarin in rodents. Regul. Toxicol. Pharmacol. 60, 106-111. doi: 10.1016/j.yrtph.2011.02.013

Lin, Q. R., Li, C. G., Zha, Q. B., Xu, L. H., Pan, H., Zhao, G. X., et al. (2016). Gossypol induces pyroptosis in mouse macrophages via a non-canonical inflammasome pathway. Toxicol. Appl. Pharmacol. 292, 56-64. doi: 10.1016/j. taap.2015.12.027

Liu, X., Zhang, Z., Ruan, J., Pan, Y., Magupalli, V. G., Wu, H., et al. (2016). Inflammasome-activated gasdermin $\mathrm{D}$ causes pyroptosis by forming membrane pores. Nature 535, 153-158. doi: 10.1038/nature18629

Long, H., Xu, B., Luo, Y., and Luo, K. (2016). Artemisinin protects mice against burn sepsis through inhibiting NLRP3 inflammasome activation. Am. J. Emerg. Med. 34, 772-777. doi: 10.1016/j.ajem.2015.12.075

Lotze, M. T., and Tracey, K. J. (2005). High-mobility group box 1 protein (HMGB1): nuclear weapon in the immune arsenal. Nat. Rev. Immunol. 5, 331-342. doi: 10.1038/nri1594

Lucarini, R., Tozatti, M. G., Silva, M. L., Gimenez, V. M., Pauletti, P. M., Groppo, M., et al. (2015). Antibacterial and anti-inflammatory activities of an extract, fractions, and compounds isolated from Gochnatia pulchra aerial parts. Braz. J. Med. Biol. Res. 48, 822-830. doi: 10.1590/1414-431x201 54410

Mariathasan, S., Weiss, D. S., Newton, K., McBride, J., O’Rourke, K., RooseGirma, M., et al. (2006). Cryopyrin activates the inflammasome in response to toxins and ATP. Nature 440, 228-232. doi: 10.1038/nature04515

Marshall, J. C. (2014). Why have clinical trials in sepsis failed? Trends Mol. Med. 20, 195-203. doi: 10.1016/j.molmed.2014.01.007

Martinon, F., Petrilli, V., Mayor, A., Tardivel, A., and Tschopp, J. (2006). Goutassociated uric acid crystals activate the NALP3 inflammasome. Nature 440, 237-241. doi: 10.1038/nature04516 
Mortimer, L., Moreau, F., MacDonald, J. A., and Chadee, K. (2016). NLRP3 inflammasome inhibition is disrupted in a group of auto-inflammatory disease CAPS mutations. Nat. Immunol. 17, 1176-1186. doi: 10.1038/ni.3538

Munoz-Planillo, R., Kuffa, P., Martinez-Colon, G., Smith, B. L., Rajendiran, T. M., and Nunez, G. (2013). $\mathrm{K}^{+}$efflux is the common trigger of NLRP3 inflammasome activation by bacterial toxins and particulate matter. Immunity 38, 1142-1153. doi: 10.1016/j.immuni.2013.05.016

Niu, C., Sheng, Y., Yang, R., Lu, B., Bai, Q., Ji, L., et al. (2015). Scutellarin protects against the liver injury induced by diosbulbin B in mice and its mechanism. J. Ethnopharmacol. 164, 301-308. doi: 10.1016/j.jep.2015.02.031

Pan, H., Xu, L. H., Huang, M. Y., Zha, Q. B., Zhao, G. X., Hou, X. F., et al. (2015). Piperine metabolically regulates peritoneal resident macrophages to potentiate their functions against bacterial infection. Oncotarget 6, 32468-32483. doi: 10.18632/oncotarget.5957

Piccini, A., Carta, S., Tassi, S., Lasiglie, D., Fossati, G., and Rubartelli, A. (2008). ATP is released by monocytes stimulated with pathogen-sensing receptor ligands and induces IL-1beta and IL-18 secretion in an autocrine way. Proc. Natl. Acad. Sci. U.S.A. 105, 8067-8072. doi: 10.1073/pnas.0709684105

Py, B. F., Jin, M., Desai, B. N., Penumaka, A., Zhu, H., Kober, M., et al. (2014). Caspase-11 controls interleukin-1beta release through degradation of TRPC1. Cell Rep. 6, 1122-1128. doi: 10.1016/j.celrep.2014.02.015

Rathinam, V. A., and Fitzgerald, K. A. (2016). Inflammasome complexes: emerging mechanisms and effector functions. Cell 165, 792-800. doi: 10.1016/j.cell.2016. 03.046

Sborgi, L., Ruhl, S., Mulvihill, E., Pipercevic, J., Heilig, R., Stahlberg, H., et al. (2016). GSDMD membrane pore formation constitutes the mechanism of pyroptotic cell death. EMBO J. 35, 1766-1778. doi: 10.15252/embj.201694696

Shaw, P. J., Lukens, J. R., Burns, S., Chi, H., McGargill, M. A., and Kanneganti, T. D. (2010). Cutting edge: critical role for PYCARD/ASC in the development of experimental autoimmune encephalomyelitis. J. Immunol. 184, 4610-4614. doi: 10.4049/jimmunol.1000217

Shi, J., Gao, W., and Shao, F. (2017). Pyroptosis: gasdermin-mediated programmed necrotic cell death. Trends Biochem. Sci. 42, 245-254. doi: 10.1016/j.tibs.2016. 10.004

Shi, J., Zhao, Y., Wang, K., Shi, X., Wang, Y., Huang, H., et al. (2015). Cleavage of GSDMD by inflammatory caspases determines pyroptotic cell death. Nature 526, 660-665. doi: 10.1038/nature15514

Singer, M., Deutschman, C. S., Seymour, C. W., Shankar-Hari, M., Annane, D., Bauer, M., et al. (2016). The third international consensus definitions for sepsis and septic shock (sepsis-3). JAMA 315, 801-810. doi: 10.1001/jama.2016.0287

Tan, Z. H., Yu, L. H., Wei, H. L., and Liu, G. T. (2010). Scutellarin protects against lipopolysaccharide-induced acute lung injury via inhibition of NF-kappaB activation in mice. J. Asian Nat. Prod. Res. 12, 175-184. doi: 10.1080/ 10286020903347906

Tian, X., Chang, L., Ma, G., Wang, T., Lv, M., Wang, Z., et al. (2016). Delineation of platelet activation pathway of scutellarein revealed its intracellular target as protein kinase C. Biol. Pharm. Bull. 39, 181-191. doi: 10.1248/bpb.b15-00511

van der Poll, T., van de Veerdonk, F. L., Scicluna, B. P., and Netea, M. G. (2017). The immunopathology of sepsis and potential therapeutic targets. Nat. Rev. Immunol. 17, 407-420. doi: 10.1038/nri.2017.36

Vandanmagsar, B., Youm, Y. H., Ravussin, A., Galgani, J. E., Stadler, K., Mynatt, R. L., et al. (2011). The NLRP3 inflammasome instigates obesity-induced inflammation and insulin resistance. Nat. Med. 17, 179-188. doi: 10.1038/nm. 2279

Wang, S., Wang, H., Guo, H., Kang, L., Gao, X., and Hu, L. (2011). Neuroprotection of Scutellarin is mediated by inhibition of microglial inflammatory activation. Neuroscience 185, 150-160. doi: 10.1016/j.neuroscience.2011.04.005

Wang, W., Ma, X., Han, J., Zhou, M., Ren, H., Pan, Q., et al. (2016). Neuroprotective effect of scutellarin on ischemic cerebral injury by down-regulating the expression of angiotensin-converting enzyme and AT1 receptor. PLOS ONE 11:e0146197. doi: 10.1371/journal.pone.0146197

Wang, Y., Yang, C., Mao, K., Chen, S., Meng, G., and Sun, B. (2013). Cellular localization of NLRP3 inflammasome. Protein Cell 4, 425-431. doi: 10.1007/ s13238-013-2113-2
Wang, Z., Yu, J., Wu, J., Qi, F., Wang, H., Wang, Z., et al. (2016). Scutellarin protects cardiomyocyte ischemia-reperfusion injury by reducing apoptosis and oxidative stress. Life Sci. 157, 200-207. doi: 10.1016/j.lfs.2016.01.018

Wegiel, B., Larsen, R., Gallo, D., Chin, B. Y., Harris, C., Mannam, P., et al. (2014). Macrophages sense and kill bacteria through carbon monoxide-dependent inflammasome activation. J. Clin. Invest. 124, 4926-4940. doi: 10.1172/jci 72853

Wen, H., Gris, D., Lei, Y., Jha, S., Zhang, L., Huang, M. T., et al. (2011). Fatty acid-induced NLRP3-ASC inflammasome activation interferes with insulin signaling. Nat. Immunol. 12, 408-415. doi: 10.1038/ni.2022

Wen, H., Ting, J. P., and O'Neill, L. A. (2012). A role for the NLRP3 inflammasome in metabolic diseases-did Warburg miss inflammation? Nat. Immunol. 13, 352-357. doi: 10.1038/ni.2228

Wree, A., Eguchi, A., McGeough, M. D., Pena, C. A., Johnson, C. D., Canbay, A., et al. (2014). NLRP3 inflammasome activation results in hepatocyte pyroptosis, liver inflammation, and fibrosis in mice. Hepatology 59, 898-910. doi: 10.1002/ hep.26592

Yan, Y., Jiang, W., Liu, L., Wang, X., Ding, C., Tian, Z., et al. (2015). Dopamine controls systemic inflammation through inhibition of NLRP3 inflammasome. Cell 160, 62-73. doi: 10.1016/j.cell.2014.11.047

Yanai, H., Ban, T., and Taniguchi, T. (2012). High-mobility group box family of proteins: ligand and sensor for innate immunity. Trends Immunol. 33, 633-640. doi: 10.1016/j.it.2012.10.005

Youm, Y. H., Nguyen, K. Y., Grant, R. W., Goldberg, E. L., Bodogai, M., Kim, D., et al. (2015). The ketone metabolite beta-hydroxybutyrate blocks NLRP3 inflammasome-mediated inflammatory disease. Nat. Med. 21, 263-269. doi: $10.1038 / \mathrm{nm} .3804$

Yuan, Y., Fang, M., Wu, C. Y., and Ling, E. A. (2016). Scutellarin as a potential therapeutic agent for microglia-mediated neuroinflammation in cerebral ischemia. Neuromol. Med. 18, 264-273. doi: 10.1007/s12017-0168394-X

Yuan, Y., Rangarajan, P., Kan, E. M., Wu, Y., Wu, C., and Ling, E. A. (2015). Scutellarin regulates the Notch pathway and affects the migration and morphological transformation of activated microglia in experimentally induced cerebral ischemia in rats and in activated BV-2 microglia. J. Neuroinflammation 12:11. doi: 10.1186/s12974-014-0226-Z

Yuan, Y., Zha, H., Rangarajan, P., Ling, E. A., and Wu, C. (2014). Antiinflammatory effects of Edaravone and Scutellarin in activated microglia in experimentally induced ischemia injury in rats and in BV-2 microglia. BMC Neurosci. 15:125. doi: 10.1186/s12868-014-0125-3

Zha, Q. B., Wei, H. X., Li, C. G., Liang, Y. D., Xu, L. H., Bai, W. J., et al. (2016). ATP-induced inflammasome activation and pyroptosis is regulated by AMP-activated protein kinase in macrophages. Front. Immunol. 7:597. doi: 10.3389/fimmu.2016.00597

Zhao, G. X., Xu, L. H., Pan, H., Lin, Q. R., Huang, M. Y., Cai, J. Y., et al. (2015). The BH3-mimetic gossypol and noncytotoxic doses of valproic acid induce apoptosis by suppressing cyclin-A2/Akt/FOXO3a signaling. Oncotarget 6, 38952-38966. doi: 10.18632/oncotarget.5731

Zhao, S., Sun, Y., Li, X., Wang, J., Yan, L., Zhang, Z., et al. (2016). Scutellarin inhibits RANKL-mediated osteoclastogenesis and titanium particle-induced osteolysis via suppression of NF-kappaB and MAPK signaling pathway. Int. Immunopharmacol. 40, 458-465. doi: 10.1016/j.intimp.2016.09.031

Conflict of Interest Statement: The authors declare that the research was conducted in the absence of any commercial or financial relationships that could be construed as a potential conflict of interest.

Copyright (c) 2018 Liu, Jing, Zeng, Li, Xu, Yan, Bai, Zha, Ouyang and He. This is an open-access article distributed under the terms of the Creative Commons Attribution License (CC BY). The use, distribution or reproduction in other forums is permitted, provided the original author(s) or licensor are credited and that the original publication in this journal is cited, in accordance with accepted academic practice. No use, distribution or reproduction is permitted which does not comply with these terms. 